\title{
Sonic Boom Prediction and Mitigation using Three-Dimensional Earth Effects
}

\author{
Sriram K. Rallabhandi* \\ NASA Langley Research Center, Hampton, Virginia, 23681
}

\begin{abstract}
As the industry progresses towards realizing a commercial supersonic aircraft, the push to reduce noise from sonic boom has intensified. This emphasizes the need to better understand and accurately model the underlying physics and seek ways to reduce modeling gaps in the calculation of sonic boom noise metrics. To answer this need, sonic boom prediction capability of the atmospheric propagation tool SBOOM is enhanced to include three-dimensional Earth effects during propagation. The discrete adjoint capability under three-dimensional effects is also enhanced to be helpful in design optimization. The differences in ground signatures, noise metrics, and carpet widths are compared against results obtained using flat Earth approximation and discussed. Finally, the developed capability is used in CFD based shape optimization to demonstrate the differences in aircraft outer mold line when using flat and ellipsoidal Earth effects during shape optimization for sonic boom mitigation.
\end{abstract}

\section{Nomenclature}

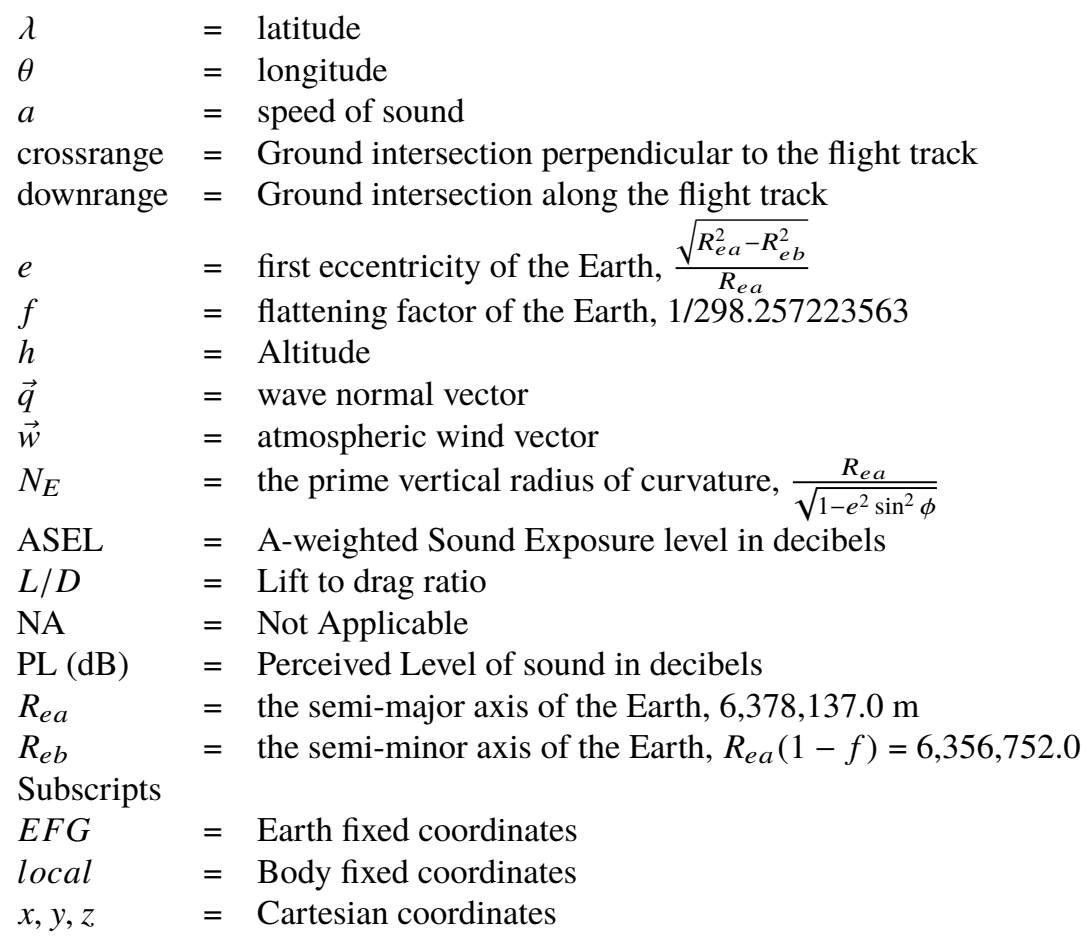

\section{Introduction and Motivation}

NASA [1-5] and industry [6-12] are moving at a fast pace to enhance simulation tools to bring a commercial Nupersonic concept to fruition. There have also been efforts [13-16] to bring awareness to the broader aerospace community on the aspects of sonic boom prediction, impact and community response. With the planned NASA

\footnotetext{
*Aerospace Engineer, Aeronautics Systems Analysis Branch, Mail Stop 442, and AIAA Associate Fellow.
} 
supersonic X-plane [17] flight demonstration process, there is an opportunity to influence public acceptability and potentially lift the band on current commercial supersonic flight over land. When that happens, there are multiple industry concepts [6, 18-20] that aim to build and introduce commercial supersonic travel at a range of supersonic cruise Mach numbers. While some companies plan to fly below the Mach cut-off [10] or avoid supersonic overland flight [18], one of the most challenging aspects is the ability to fly at supersonic speeds overland with minimal community impact. As we move to business jets and even larger passenger capacity supersonic concepts, there is a need to constantly enhance sonic boom prediction and design tools to model the primary metrics of interest with ever increasing fidelity.

Multiple efforts have been undertaken in the past several years that have contributed to and enhanced the sonic boom prediction capability. These include enhancements in near-field pressure waveform modeling using Computational Fluid Dynamics (CFD) as well as atmospheric propagation. For near-field pressure prediction, recent lessons learned include the need to run viscous as opposed to inviscid simulations to extract more accurate pressure information, resolving plume effects [21], and inlet integration [22]. Atmospheric propagation also has seen enhancements in the form of lossy propagation [23], adjoint methods [3, 24], non-empirical physics-based focus prediction [25], edge of the boom carpet diffraction [26], and atmospheric turbulence [27] effects. However, several other effects such as spherical or ellipsoid Earth effects, mean-flow wind effects [28, 29], prediction in the shadow region [26], over-the-top secondary booms etc., have not been extensively researched, particularly for lossy propagation implementations, though formulations exist in literature. This paper attempts to include ellipsoidal Earth effects in the context of lossy atmospheric propagation and document the differences with results from traditional flat Earth models. Inclusion of such effects in ray path calculations is not unique; other implementations [30] have this feature, but perhaps only for lossless propagation. The paper, however, demonstrates, perhaps for the first time in literature, the effect of ellipsoidal Earth effects on shape optimization of a notional aircraft and discusses the differences observed with and without flat Earth assumption during atmospheric propagation in the context of aircraft design for sonic boom mitigation.

The rest of the paper is organized as follows. Section $\Pi$ presents the methodology used in extending the ray paths for ellipsoidal Earth effects. Section [III presents the discrete-adjoint sensitivities and their verification using the complex-step approach. Section [V] presents the results of the high fidelity shape optimization to reduce sonic boom in the presence of three dimensional Earth effects, and discusses the impact of the capability as well as the optimization. Finally, section $\mathrm{V}$ provides conclusions based on the work conducted.

\section{Methodology}

Most sonic boom prediction studies approximate the Earth as a flat surface when predicting the impact at the ground level. This is perhaps acceptable for primary boom carpet where the disturbances from the aircraft are propagating toward the ground and the propagation distances are small relative to the Earth's curvature. However, for secondary booms (both ground reflections and over-the-top propagation) and for primary booms with large winds, propagation distances may not be small compared to the Earth's curvature, and thus cannot be ignored. Ray tracing needs to be enhanced to be carried out in Earth fixed geocentric (EFG) coordinate system. This means that the aircraft-based coordinate system needs to be transformed into EFG coordinates. Figure 1 depicts the Earth fixed geocentric (EFG) and Geodetic coordinate systems. The aircraft location in terms of both EFG (Cartesian) coordinates and Geodetic coordinates [latitude $(\lambda)$, longitude $(\theta)$ and altitude $(h)$ ] are depicted. The methods presented in [31, 32] are utilized to carry out the necessary coordinate transformations. Specifically, Eq. (1) is used to transform local coordinates into EFG coordinates using the position vector in the Geodetic frame.

$$
\left[\begin{array}{c}
X_{E F G} \\
Y_{E F G} \\
Z_{E F G}
\end{array}\right]=\left[\begin{array}{ccc}
-\sin \lambda \cos \theta & -\sin \theta & \cos \lambda \cos \theta \\
-\sin \theta \sin \lambda & \cos \theta & \sin \theta \cos \lambda \\
\cos \lambda & 0 & \sin \lambda
\end{array}\right]\left[\begin{array}{c}
X_{\text {local }} \\
Y_{\text {local }} \\
Z_{\text {local }}
\end{array}\right]
$$

While no closed form solutions exist to convert EFG coordinates to Geodetic coordinates, an iterative procedure laid out in Ref. [32] is used in this study. Other similar techniques exist in literature [33, 34] to perform this conversion. The conversion from Geodetic to EFG frame is accomplished using Eq. (2), where e and $N_{E}$ are first eccentricity and the prime vertical radius of curvature of the Earth respectively. 


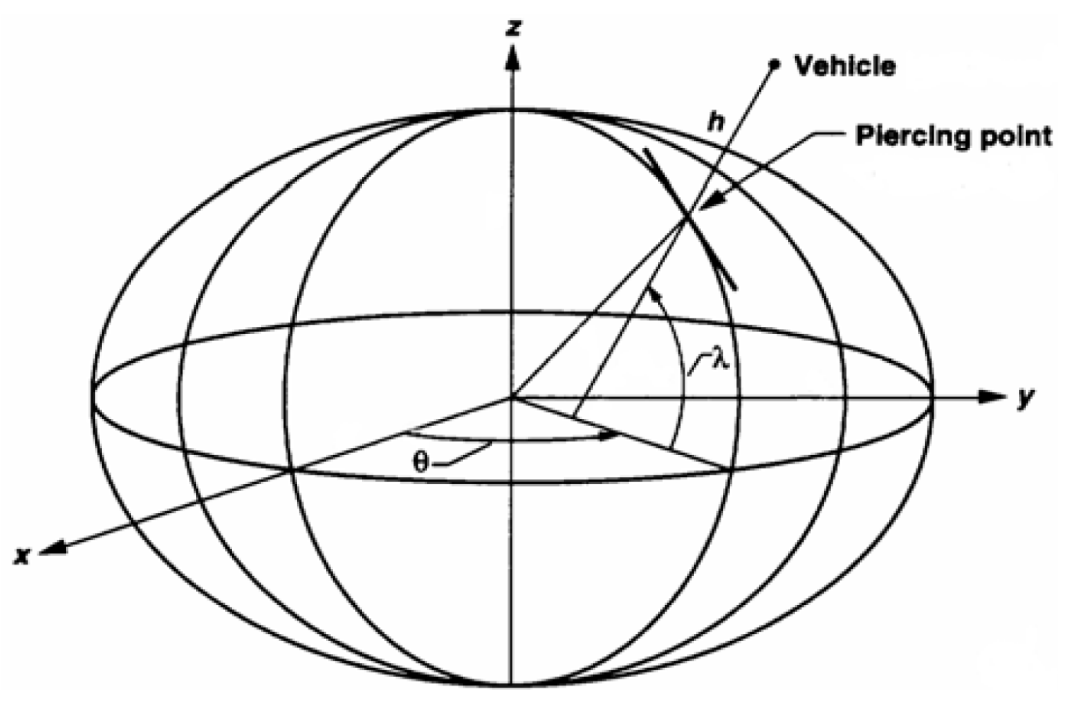

Fig. 1 Ellipsoidal Earth [30]

$$
\begin{array}{r}
X_{E F G}=\left(N_{E}+h\right) \cos \lambda \cos \theta \\
Y_{E F G}=\left(N_{E}+h\right) \cos \lambda \sin \theta \\
Z_{E F G}=\left(N_{E}\left[1-e^{2}\right]+h\right) \sin \lambda
\end{array}
$$

Once the coordinate transformations are in place, three dimensional ray path integration is based on the method presented by Schulten [35]. Equation (3) specifies the ray tracing equations, where $\vec{q}$ is the wave normal vector, $a$ is the speed of sound, and $\vec{w}$ is the atmospheric wind velocity vector. Eq. (4) is the Cartesian component equivalent of Eq. (3). While just the vertical gradients are needed in the flat Earth schemes, in the three-dimensional ellipsoid Earth formulation, derivatives of the prevailing wind and speed of sound gradients are required with respect to all three axes. The atmospheric propagation code sBOOM [36] is used as the basis for atmospheric propagation. Numerical modeling is based on lossy Burgers' Equation solver that takes into effect absorption and relaxation losses during propagation.

$$
\begin{gathered}
\frac{d \vec{q}}{d t}=-|\vec{q}| \nabla a-\vec{q} \times(\nabla \times \vec{w})-(\vec{q} \cdot \nabla) \vec{w} \\
\frac{d q_{x}}{d t}=-|\vec{q}| \frac{\partial a}{\partial x}-\vec{q} \cdot \frac{\partial \vec{w}}{\partial x} \\
\frac{d q_{y}}{d t}=-|\vec{q}| \frac{\partial a}{\partial y}-\vec{q} \cdot \frac{\partial \vec{w}}{\partial y} \\
\frac{d q_{z}}{d t}=-|\vec{q}| \frac{\partial a}{\partial z}-\vec{q} \cdot \frac{\partial \vec{w}}{\partial z}
\end{gathered}
$$

Figure 2 depicts the comparison of the ray paths for the flat Earth mode, and ellipsoid Earth mode for the latitudes of 0 and 70 degrees in the absence of wind. For these cases, there is visually no difference in the ray paths between the flat and ellipsoidal Earth propagation modes. The small differences in the ray paths are also quantitatively seen in Table 11 where, compared to the propagation using the flat earth mode, the downrange and crossrange are within $1 \%$. Even for the crossrange cases with percentage differences of $\tilde{1} \%$ with respect to the flat earth propagation distances, the actual magnitude differences are quite small.

While secondary booms might have a larger impact due to Earth's curvature, this work focuses on primary booms. To look for differences in primary booms in the presence of winds, the Integrated Global Radiosonde Archive (IGRA) [37] database, consisting of radiosonde balloon observations including temperature, pressure, dew point (a proxy for relative humidity), and winds at several active sites was explored. Multiple, geographically spread locations around the 


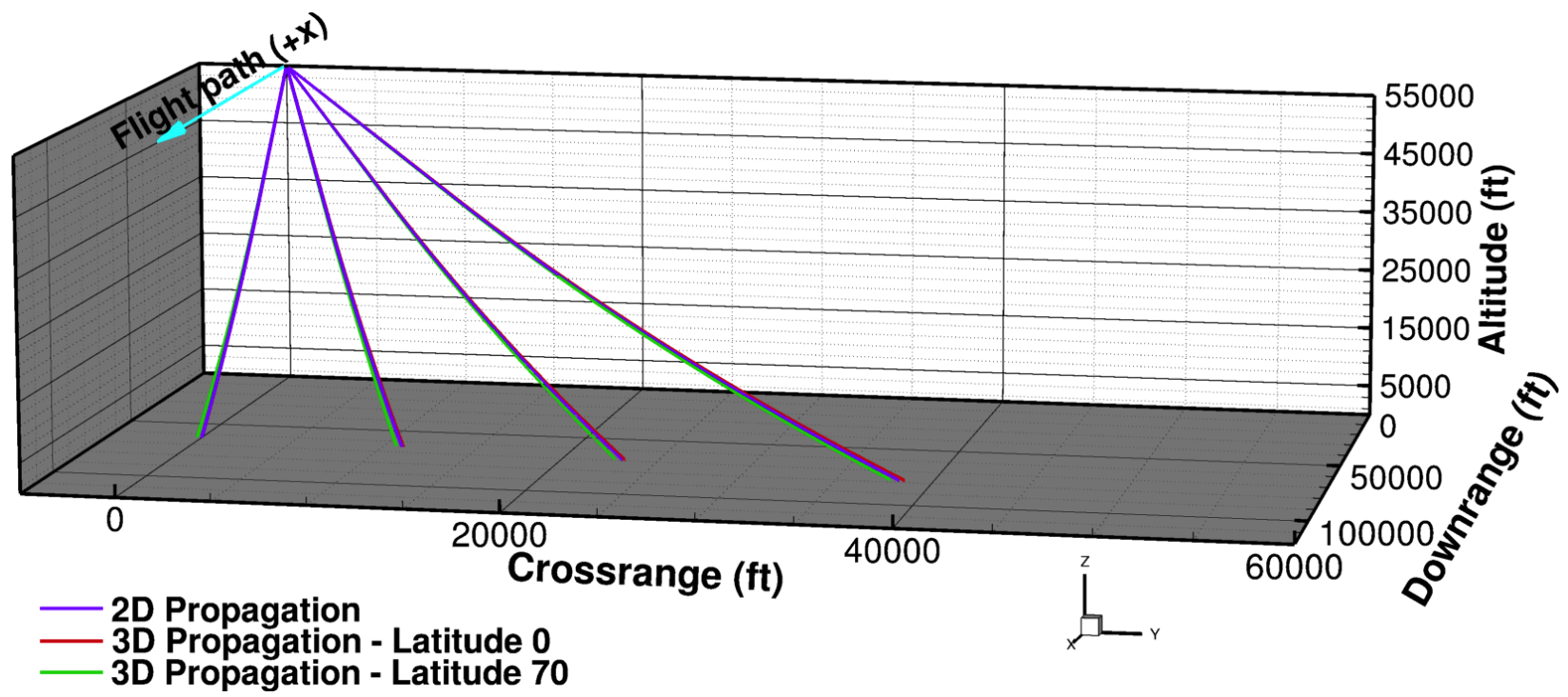

Fig. 2 Comparison of ray paths under zero winds

Table 1 Comparison of ray paths under idealized no wind conditions

\begin{tabular}{ccccc}
\hline Ellipsoidal Earth? & Roll Angle $\left(^{o}\right)$ & Latitude $\left(^{o}\right)$ & Downrange,Kft.(Diff\%) & Crossrange,Kft. (Diff\%) \\
\hline No & 0 & NA & 63.33 (NA) & 0.0 (NA) \\
Yes & 0 & 0 & $63.28(-0.08)$ & $4.28 \mathrm{e}-05$ (NA) \\
Yes & 0 & 70 & $63.10(-0.36)$ & -0.27 (NA) \\
\hline No & 30 & NA & $77.93($ NA) & 38.17 (NA) \\
Yes & 30 & 0 & $78.00(0.09)$ & $38.47(0.79)$ \\
Yes & 30 & 70 & $77.78(-0.19)$ & $37.72(-1.17)$ \\
\hline
\end{tabular}

world were searched for atmospheric profiles with high winds, and one was selected that had the highest zonal wind. Figure 3 depicts the atmospheric profile chosen for the wind case. Large magnitude winds, upwards of 80 meters/sec at certain altitudes, are observed for this profile. Figure 4 shows the differences in the ray paths between the flat Earth mode and curved Earth mode. Large differences in the ground intersection locations as well as the ray paths are observed, even for the case of zero latitude. This is quantified in Table 2, where large percentage differences in ray path distances with respect to those using flat earth assumption are observed for both downrange and crossrange. This shows that even for primary booms, if the prevailing winds are large, flat Earth assumption may be inadequate for atmospheric propagation to predict sonic boom ground signatures.

Figures 5 and 6 depict the ground signatures at multiple azimuthal angles for the no-wind and measured wind case, respectively. As expected from the ray paths, for the idealized zero wind cases, the ground signatures display minor differences. However, for the measured high wind case chosen, the differences are appreciable. Figures 7 and 8 show a similar trend for the loudness metrics [38, 39]..

\section{Discrete Adjoint Sensitivities and Verification}

In addition to sonic boom prediction in ellipsoidal Earth mode, this paper also enhances the discrete-adjoint [40] capability to aid in design optimization of aircraft concepts. Sensitivities of multiple objectives, such as the A-weighted sound exposure level (ASEL) of the ground signature, with respect to the near-field waveform are obtained in addition to predicting the ground signatures and associated loudness metrics. A complex variable version of the atmospheric propagation with ellipsoidal Earth effects has been developed to verify the accuracy of the loudness sensitivity that 


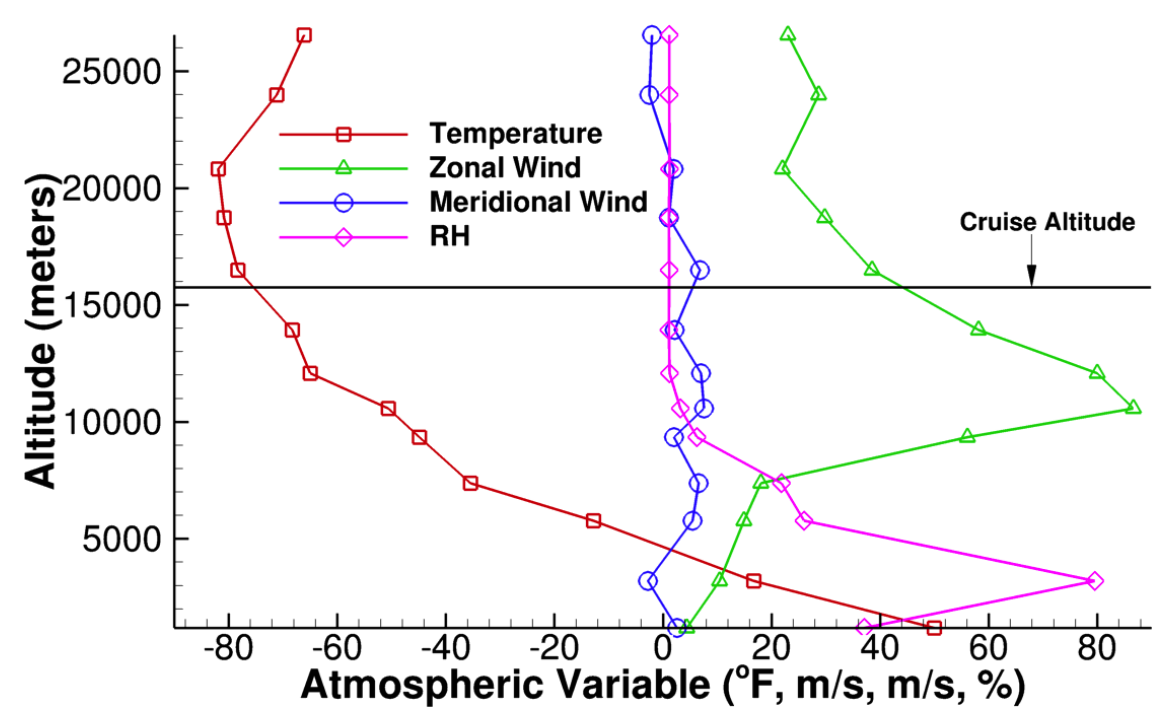

Fig. 3 Atmospheric profile chosen for comparisons

Table 2 Comparison of ray paths with arbitrary winds

\begin{tabular}{ccccc}
\hline Ellipsoidal Earth? & Roll Angle $\left(^{o}\right)$ & Latitude $\left(^{o}\right)$ & Downrange,Kft.(Diff\%) & Crossrange,Kft. (Diff\%) \\
\hline No & 0 & NA & $71.55(\mathrm{NA})$ & $-1.03(\mathrm{NA})$ \\
Yes & 0 & 0 & $59.33(-17.07)$ & $10.16(1086.41)$ \\
Yes & 0 & 70 & $75.53(5.56)$ & $2.97(388.34)$ \\
\hline No & 30 & NA & $90.65(\mathrm{NA})$ & $36.13(\mathrm{NA})$ \\
Yes & 30 & 0 & $73.03(-19.44)$ & $49.47(36.92)$ \\
Yes & 30 & 70 & $111.79(23.32)$ & $58.61(62.22)$ \\
\hline
\end{tabular}

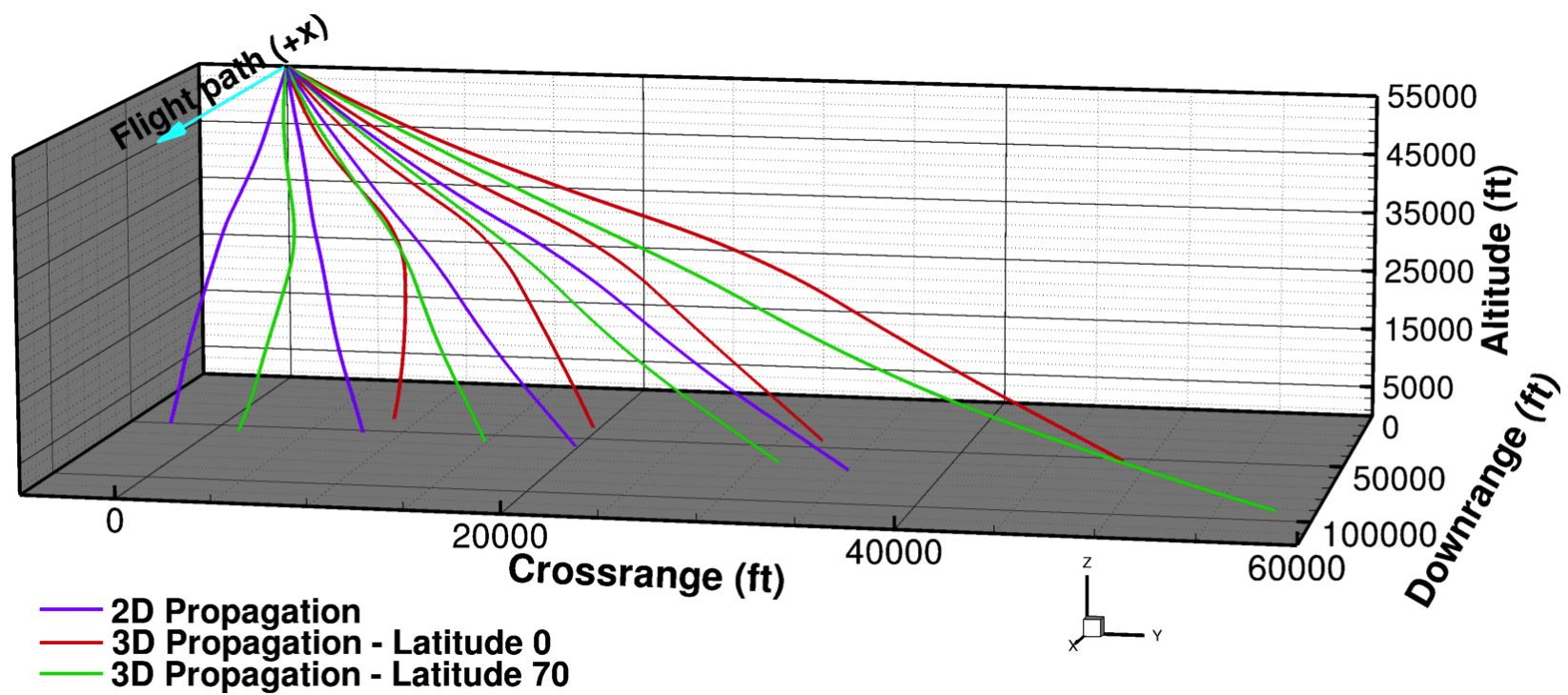

Fig. 4 Comparison of ray paths under arbitrary winds 
is calculated using the adjoint methodology. The complex variable approach [41, 42] has been applied in several other gradient verifications. The main advantage of the complex variable method is that true second-order accuracy is achieved by selecting step sizes without incurring subtractive cancellation errors typically present in real-valued finite differences. Table 3 shows the comparison of the loudness sensitivity values computed using the discrete-adjoint version and the complex version of the code for arbitrarily selected indices in the near-field waveform using an imaginary step size of $10^{-50}$. From these results, it can be seen that sensitivity values match up to 10 decimal places, thus verifying that the sonic boom loudness sensitivity values being computed with ellipsoidal Earth effects are accurate.

Table 3 Comparison of adjoint and complex variable gradients with Ellipsoidal Earth effects

\begin{tabular}{ccc}
\hline Grid Point & Complex Variable Gradient & Adjoint Gradient \\
\hline 1 & 40.446126133717763 & $40.4461261337 \underline{24860}$ \\
100 & 27.327694119379854 & $27.3276941193 \underline{\underline{53189}}$ \\
200 & 10.528727698107521 & $10.5287276981 \underline{\underline{90500}}$ \\
400 & 29.446653633109131 & $29.44665363310 \underline{08489}$ \\
600 & -11.988688512771946 & $-11.9886885127 \underline{51837}$ \\
\hline
\end{tabular}

Figures 9 and 10 depict a sample near-field off-body pressure waveform overlaid with ASEL sensitivities of the sonic boom ground signature for the idealized no wind case and the high wind case respectively. As has been observed earlier for ray paths and ground signatures, the ASEL sensitivities in the zero wind case for the flat Earth and ellipsoidal Earth cases are virtually identical. However, for the high wind cases, large differences are observed, especially at higher off-track azimuthal angles. Since loudness sensitivities are used in shape optimization to drive the underlying design toward lower sonic boom levels, it is expected that there will be differences in the shape optimization results when using flat Earth and ellipsoidal Earth effects. This is demonstrated in the next section.

\section{Impact of Ellipsoidal Earth Propagation Effects on Shape Optimization}

The shape optimization setup used in this study is identical to that presented in an earlier study [3]. This was done to minimize the effort to set up a new case as well as to quickly demonstrate the developed capability; however the shape optimization is applicable to any other concept that needs to be optimized. Interested readers should refer to Ref. [3] for further details on the parameterization scheme used and other details of the problem setup. For completeness, relevant details are provided here briefly. The aircraft concept being optimized is shown in Fig. 11, which is a variant of the concept presented in Refs. [4, 5]. This baseline configuration is the result of earlier optimization using mixed-fidelity [43], reversed-equivalent-area [44] methods. This baseline configuration has also been successfully optimized in earlier work [4, 5] using Cart3D [2] and flat Earth assumption for atmospheric propagation. The initial mesh for this concept was generated using VGRID [45], and SSGRID [46].

The CFD grid utilizes a plane of symmetry along the centerline and contains 8.5 million nodes, 15 million tetrahedral elements and 11 million prisms. The surface mesh for the aircraft is parameterized using a free-form shape deformation tool called BANDAIDS [47]. All the components of the baseline, except the inner cowl of the flow-through nacelles, are parameterized and allowed to vary in the shape optimization exercise. The intersections between aircraft components are held fixed for simplicity, although this is not a requirement of the formulation. The parameters for shape modification are NURBS control points. However, to make smooth changes to the underlying mesh, multiple control points are grouped for the optimizer to modify simultaneously. After such control point groupings, there are a total of 91 design variables for modifying the different components of the aircraft concept, but only 74 of them are allowed to change during optimization. Of these 74 design variables, 25 are used on the fuselage, 16 on wing, 7 on vertical tail, 14 on horizontal tail and 12 on the outer nacelle. The primary criteria in the choice of parameterization was to strike a balance between number of design variables and deformation smoothness. There may exist other parameterizations that satisfy these criteria and may work better, but were not tried in this study.

Shape optimization was attempted with flat Earth mode as well as ellipsoidal Earth mode for latitudes of 0 and 70 degrees (labeled 2D_Opt, 3D_0_Opt and 3D_70_Opt respectively) using the high wind atmospheric profile from section III. The objective of the optimization was minimization of ASEL corresponding to the sonic boom ground signature along the flight track, while maintaining an $L / D$ greater than or equal to the baseline value. Fig. 12 depicts 
the baseline pressure waveform along with the optima obtained using flat and ellipsoidal Earth effects for a couple of latitudes. Fig. 13 depicts the ground signatures corresponding to each of the cases. In all the optimizations, a reduction of around $5 \mathrm{~dB}$ is observed in the ASEL metric. While perceived level [38] of the sonic boom was not used in the optimization, they were computed for the optimum shapes to be $74.25 \mathrm{~dB}, 74.1 \mathrm{~dB}$ and $72.0 \mathrm{~dB}$ for the flat Earth, ellipsoidal Earth at 0 and 70 degrees respectively, compared to the baseline value of $77.6 \mathrm{~dB}$.

In a practical scenario, the designer would re-parameterize the optimum shape based on the sensitivity contours, and re-optimize until further improvements cannot be achieved. However, since this is an academic exercise with the sole purpose being demonstration of the atmospheric propagation effects of ellipsoidal Earth and its corresponding shape optimization, those steps are avoided here and conclusions are drawn based on the results obtained after this first phase of the shape optimization.

In the case of optimization with ellipsoidal Earth effects, a lower ASEL optimum is obtained, most likely due to the larger ray paths as the Earth's curvature provides a relieving effect. This effect is observed to be larger for higher latitudes. Figs. 14, 15 and 16 depict the normal displacement contours of the optimum outer-mold-line (OML) with respect to the baseline. The normal displacements displayed are in feet, showing that the optimizer makes changes on the order of a few millimeters to realize adequate gains in ground signature loudness levels. Another important observation is that there are subtle differences in the optimum shape generated by each optimization run showing that multiple highly correlated, but distinct concept geometries exist that can reduce the sonic boom loudness depending on the propagation conditions.

Finally, Figs. 17, 18, and 19 depict the surface sensitivity contours of ground-based objective function with respect to the normal perturbations to the surface geometry for the baseline and optimal configurations. As expected for under-track boom metrics, the lower surface of the aircraft is more sensitive than the upper surface. The adjoint process attempts to make the design parameters associated with the active design variables insensitive to the objective function as evidenced by the larger fraction of green regions in the sensitivity contour spectrum for the optimum design. Overall, the top surface of the optimal configuration is much less sensitive to the objective than the baseline. Certain locations on the lower side of the optimal configuration have larger sensitivities than the baseline either because changes in those locations would violate the imposed design constraints or they are closer to the component intersections that have been deliberately frozen to keep the parameterization simple or due to the underlying parameterization being inadequate. This is useful information to the designer as it suggests that further reduction of loudness is possible either by allowing component intersections to vary or by updating the parameterization. Ideally, the designer could use this information to update the parameterization and restrict the active design variables to have the highest impact on the objective. These help choose appropriate design variables in the shape optimization process or during design iterations when re-parametrization may be needed. As mentioned previosuly, these steps are avoided here but are crucial in actual design studies for extracting maximum performance from the design tools.

This paper presented extensions to the sonic boom prediction code sBOOM to account for ellipsoid Earth effects during propagation. While the formulation is not new, the computation of discrete-adjoint sensitivities in the context of ellipsoidal Earth effects is a new addition in the literature. The impact of these sensitivities in the context of shape optimization for sonic boom mitigation has been successfully demonstrated.

As previously mentioned in the paper, primary sonic boom carpets generally are not impacted by ellipsoid Earth effects because propagation distances are smaller compared to the radius of the Earth. While this paper demonstrated that for cases with large winds primary boom carpets do vary appreciably from those using flat Earth models, the benefit of adding this functionality will be more significant when enhancements are made within sBOOM for over-the-top-boom analysis or for reflected booms that tend to have large propagation distances. Prediction of these secondary booms will be pursued in future efforts and enhancements.

\section{Conclusions}

Sonic boom prediction functionality within SBOOM has been enhanced to include ellipsoidal Earth effects in the ray path calculations. To aid in design, discrete-adjoint computations have been enhanced as well to allow computation of design sensitivities. The computed loudness metrics and carpet widths are compared with those obtained from 2-D ray path calculations. For primary boom carpets in this study, the results assuming flat Earth are very close to ellipsoidal Earth computations in the absence of winds. However, in the presence of winds, as is the case for prevailing atmospheric conditions, appreciable differences in loudness metrics and carpet widths are observed. Even within the ellipsoidal computations, sonic boom loudness metrics at lower latitudes are closer to flat Earth computations while those at larger latitudes show measurable differences as the Earth's curvature causes the ray paths to travel longer distances before 
hitting the ground.

To demonstrate the impact of ellipsoidal Earth effects, the developed capability was utilized to perform shape optimization under prescribed geometric constraints. Starting from the same baseline, using ellipsoidal Earth effects leads to lower optimum sonic boom loudness metrics as well as differences in the optimum aircraft OML. This is the case even for aircraft flying at the equator. Therefore, in the absence of atmospheric turbulence effects, loudness metrics computed using flat Earth assumption may be conservative when compared against those obtained using ellipsoidal Earth effects primarily due to the relieving effect of the third dimension providing increased area or volume for the disturbances to make an impact.

\section{Acknowledgments}

This work is supported by the NASA Advanced Air Vehicles Program, Commercial Supersonic Technology (CST) Project.

\section{References}

[1] Park, M. A., Campbell, R. L., Elmiligui, A. A., Cliff, S. E., and Nayani, S., "Specialized CFD Grid Generation Methods for Near-Field Sonic Boom Prediction,” AIAA Paper 2014-0115, 2014.

[2] Aftosmis, M., Nemec, M., and Cliff, S., “Adjoint-based Low-Boom Design with Cart3D (Invited),” AIAA Paper 2011-3500, 2011.

[3] Rallabhandi, S. K., Nielsen, E. J., and Diskin, B., "Sonic Boom Mitigation Through Aircraft Design and Adjoint Methodology," AIAA Journal of Aircraft, Vol. 51, No. 2, 2014. doi:10.2514/1.C032189.

[4] Wintzer, M., Ordaz, I., and Fenbert, J. W., "Under-track CFD-based Shape Optimization for a Low-Boom Demonstrator Concept," AIAA Paper 2015-2260, 2015.

[5] Ordaz, I., Wintzer, M., and Rallabhandi, S. K., "Full-Carpet Design of a Low-Boom Demonstrator Concept," AIAA Paper 2015-2261, 2015.

[6] Howe, D., Simmons, F., and Freund, D., "Development of the Gulfstream Quiet Spike TM for Sonic Boom Minimization," AIAA Paper 2008-0124, 2008.

[7] Magee, T. E., Shaw, S. G., and Fugal, S. R., "Experimental Validations of a Low-Boom Aircraft Design," AIAA Paper 2013-0646, 2013.

[8] Morgenstern, J. M., Buonanno, M., and Nordstrud, N., "N+2 Low Boom Wind Tunnel Model Design and Validation,” AIAA Paper 2012-3217, 2012.

[9] Tracy, R. R., Chase, J. D., and Kroo, I., "Tail-braced wing aircraft and configurations for achieving long supersonic range and low sonic boom," US Patent No. 6857599, Aerion Corporation, Feb. 2005.

[10] Liebhardt, B., Lütjens, K., Tracy, R. R., and Haas, A. O., "Exploring the Prospect of Small Supersonic Airliners - A Business Case Study Based on the Aerion AS2 Jet," AIAA Paper 2017-3588, 2017.

[11] Honda, M., and Yoshida, K., "D-SEND Project for Low Sonic Boom Design Technology," 28th International Congress of the Aeronautical Sciences (ICAS), ICAS Paper 2012-1.2.1, 2012.

[12] Yamashita, R., and Suzuki, K., "Full-Field Sonic Boom Simulation in Stratified Atmosphere,” AIAA Paper 2014-2269, 2014. doi:10.2514/6.2014-2269.

[13] Park, M. A., Aftosmis, M. J., Campbell, R. L., Carter, M. B., Cliff, S. E., and Bangert, L. S., "Summary of the 2008 NASA Fundamental Aeronautics Program Sonic Boom Prediction Workshop," AIAA Journal of Aircraft, Vol. 51, No. 3, 2014, pp. 987-1001. doi:10.2514/1.C032589.

[14] Aftosmis, M. J., and Nemec, M., "Cart3D Simulations for the First AIAA Sonic Boom Prediction Workshop,” AIAA Paper 2014-558, 2014.

[15] Anderson, G. R., Aftosmis, M. J., and Nemec, M., "Cart3D Simulations for the Second AIAA Sonic Boom Prediction Workshop," AIAA Paper 2017-3255, 2017. doi:10.2514/6.2017-3255. 
[16] Rallabhandi, S. K., and Loubeau, A., "Propagation Summary of the Second AIAA Sonic Boom Prediction Workshop," AIAA Paper No. 2017-3257, 2017.

[17] NASA Awards Contract to Build Quieter Supersonic Aircraft, 2018 (accessed April 3, 2018). URL https://www .nasa.gov/ press-release/nasa-awards-contract-to-build-quieter-supersonic-aircraft

[18] Boom Supersonic, 2018 (accessed October 23, 2017). URL https://boomsupersonic.com

[19] Aerion Corporation, 2018 (accessed October 23, 2017). URL https://www . aerionsupersonic.com

[20] Spike Aerospace Corporation, 2018 (accessed October 23, 2017). URL http://www. spikeaerospace.com

[21] Wintzer, M., Ordaz, I., and Fenbert, J. W., "Under-Track CFD-Based Shape Optimization for a Low-Boom Demonstrator Concept," AIAA Paper 2015-2260, 2015.

[22] Heath, C. M., Slater, J. W., and Rallabhandi, S. K., "Inlet Trade Study for a Low-Boom Aircraft Demonstrator," AIAA Paper 2016-4050, 2016.

[23] Rallabhandi, S. K., "Advanced Sonic Boom Prediction Using the Augmented Burgers Equation," AIAA Journal of Aircraft, Vol. 48, No. 4, 2011, pp. 1245-1253. doi:10.2514/1.C031248.

[24] Rallabhandi, S. K., "Application of Adjoint Methodology to Supersonic Aircraft Design Using Reversed Equivalent Areas," AIAA Journal of Aircraft, Vol. 51, No. 5, 2014. doi:10.2514/1.C032518.

[25] Salamone, J., Sparrow, V., Plotkin, K., and Cowart, R., "SCAMP: solution of the Lossy Nonlinear Tricomi Equation for Sonic Boom Focusing," AIAA Paper 2013-0935, 2013.

[26] Coulouvrat, F., "Sonic boom in the shadow zone: A geometrical theory of diffraction," Proceedings of Meetings on Acoustics, Vol. 111, No. 499, 2002. doi:10.1121/1.1371973.

[27] Coulouvrat, F., Luquet, D., and Marchiano, R., "Numerical model of sonic boom in 3D kinematic turbulence," AIP Conference Proceedings, Vol. 1685, 2015. doi:10.1063/1.4934474.

[28] Salamone, J., "A sonic boom propagation model including mean flow atmospheric effects," AIP Conference Proceedings, Vol. 1474, No. 1, 2012. doi:10.1063/1.4749358.

[29] Rallabhandi, S. K., "Mean Flow Atmospheric Effects and their Impact on Sonic Boom Propagation,” 2018.

[30] Page, J. A., Plotkin, K. J., and Wilmer, C., "PCBoom Version 6.6 Technical Reference and User Manual,” Wyle Report WR 10-10, Arlington, VA, December 2010.

[31] Cai, G., Chen, B. M., and Lee, T. H., Unmanned Rotorcraft Systems, Springer-Verlag, 2011, Chap. Coordinate Systems and Transformations.

[32] Clynch, J. R., Geodetic Coordinate Conversions, 2006 (accessed April 3, 2018). URL/clynchg3c. com/Technote/geodesy/ coordcvt.pdf

[33] Sofair, I., "Improved Method for Calculating Exact Geodetic Latitude and Altitude," Journal of Guidance, Control and Dynamics, Vol. 20, No. 4, 1997, pp. 824-826.

[34] Sofair, I., "Improved Method for Calculating Exact Geodetic Latitude and Altitude Revisited," Journal of Guidance, Control and Dynamics, Vol. 23, No. 2, 2000, pp. 369-369.

[35] Schulten, J. B. H. M., "Computation of Aircraft Noise Propagation Through the Atmospheric Boundary Layer," NLR TP 97-374, Amsterdam, The Netherlands, December 1997.

[36] Rallabhandi, S. K., “Advanced Sonic Boom Prediction Using Augmented Burger's Equation,” Journal of Aircraft, Vol. 48, No. 4, 2011, pp. 1245-1253.

[37] Durre, I., Vose, R. S., and Wuertz, D. B., "Overview of the Integrated Global Radiosonde Archive," Journal of Climate, Vol. 19, 2006, pp. 53-68.

[38] Stevens, S. S., "Perceived Level of Noise by Mark VII and Decibels (E)," Journal of the Acoustical Society of America, Vol. 51, No. 2B, 1972, pp. 575-601. doi:10.1121/1.1912880. 
[39] Loubeau, A., Naka, Y., Cook, B. G., Sparrow, V. W., and Morgenstern, J. M., "A New Evaluation of Noise Metrics for Sonic Booms Using Existing Data," 2nd International Sonic Boom Forum, 20th International Symposium on Nonlinear Acoustics, 2015 .

[40] Rallabhandi, S. K., “Sonic Boom Adjoint Methodology and its Applications (Invited),” AIAA Paper No. 2011-3497, 2011.

[41] Lyness, J. N., "Numerical Algorithms Based on the Theory of Complex Variables," Proceedings of the ACM 22nd National Conference, 1967, pp. 124-134.

[42] Lyness, J. N., and Moler, C. B., "Numerical Differentiation of Analytic Functions," SIAM Journal on Numerical Analysis, Vol. 4, 1967, pp. 202-210.

[43] Li, W., Shields, E., and Geiselhart, K., “A Mixed- Fidelity Approach for Design of Low- Boom Supersonic Aircraft,” AIAA Paper No. 2010-0845, 2010.

[44] Li, W., and Rallabhandi, S. K., "Inverse Design of Low-Boom Supersonic Concepts Using Reversed Equivalent-Area Targets (Invited),” AIAA Paper No. 2011-3498, 2011.

[45] Pirzadeh, S., "Three-Dimensional Unstructured Viscous Grids by the Advancing-Layers Method," AIAA Journal, Vol. 34, No. 1, 1996, pp. 43-49.

[46] Campbell, R. L., Carter, M. B., Deere, K. A., and Waithe, K. A., "Efficient Unstructured Grid Adaptation Methods for Sonic Boom Prediction," AIAA Paper 2008-7327, 2008.

[47] Samareh, J. A., “Aerodynamic Shape Optimization based on free-form deformation,” AIAA Paper 2004-4630, 2004. 

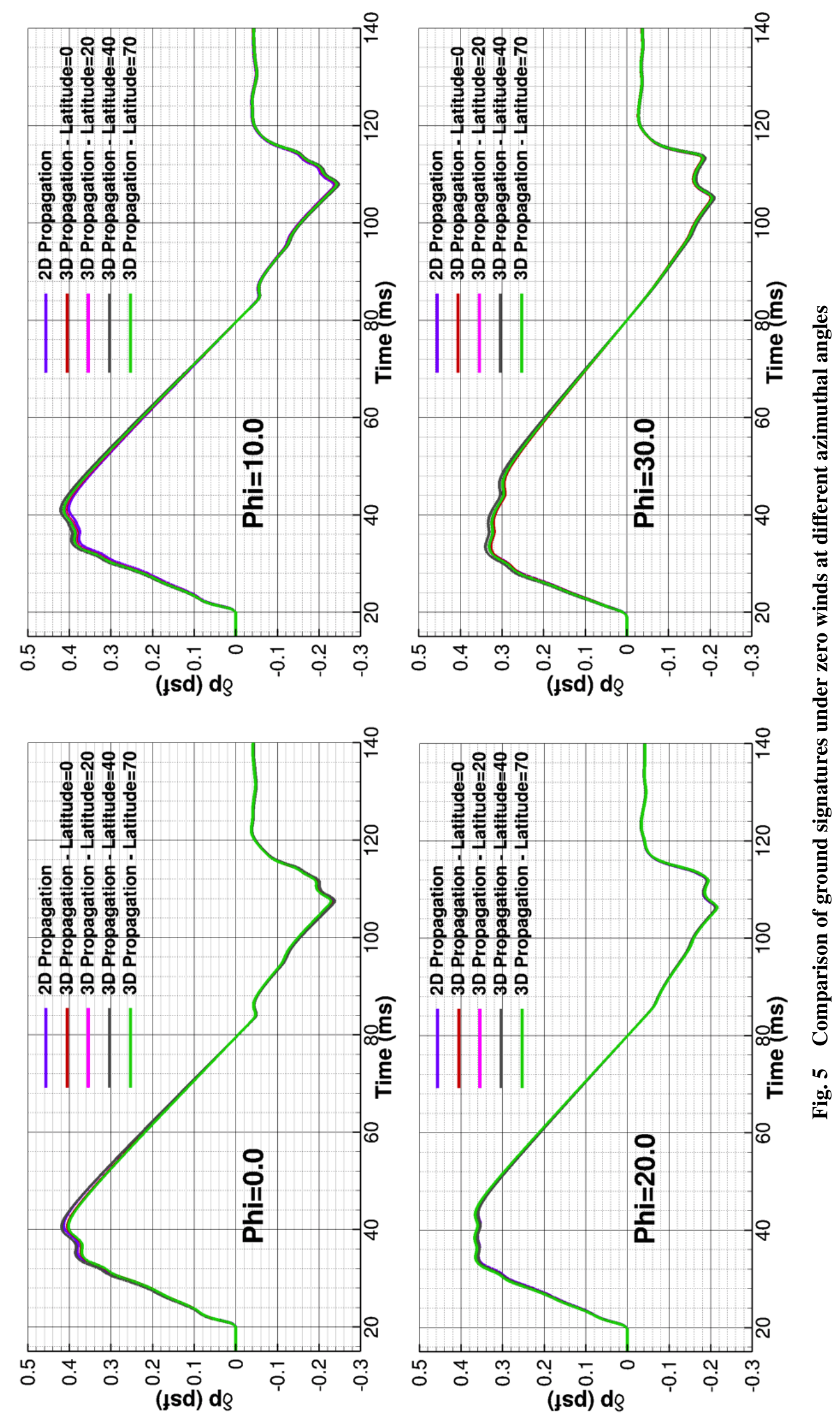

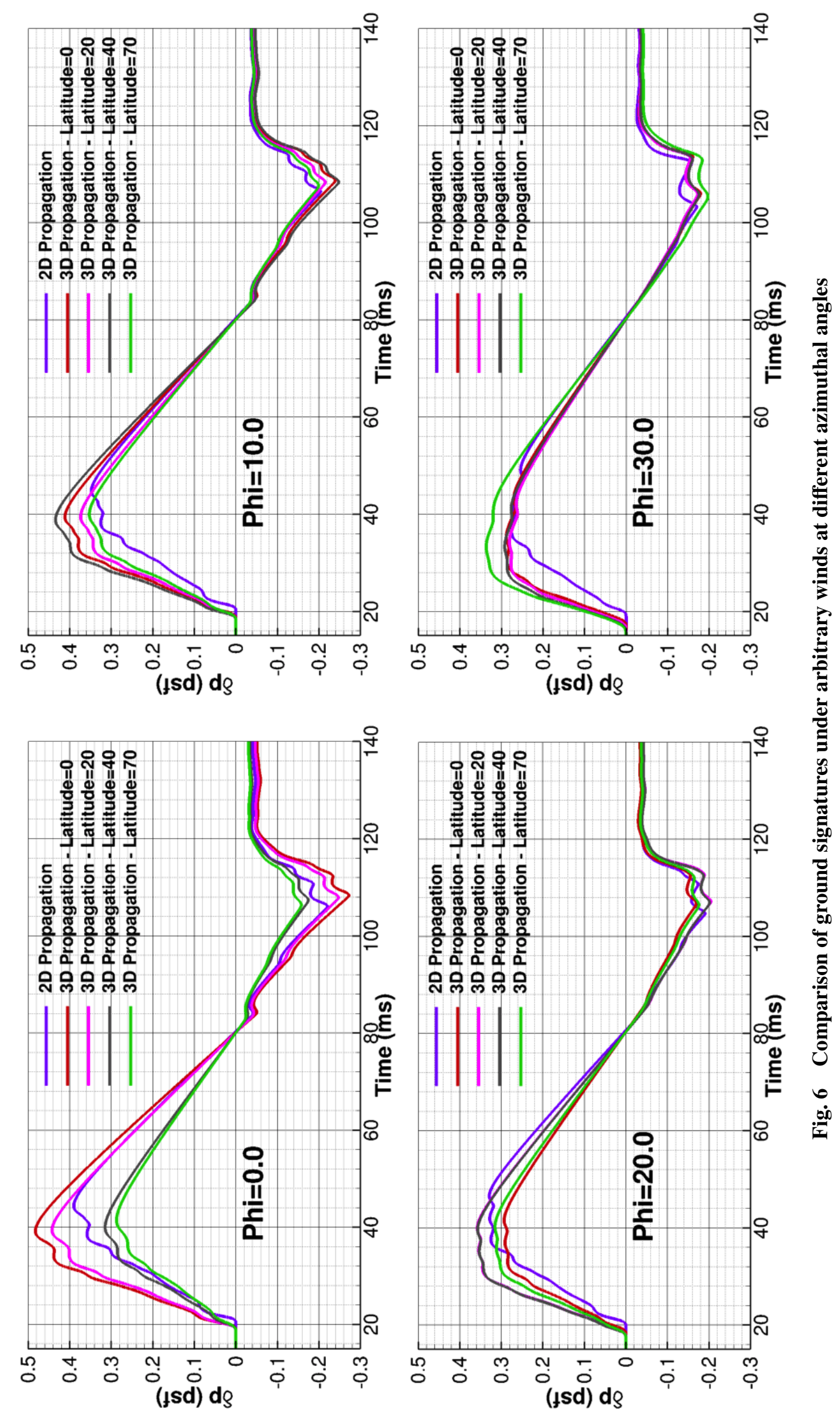


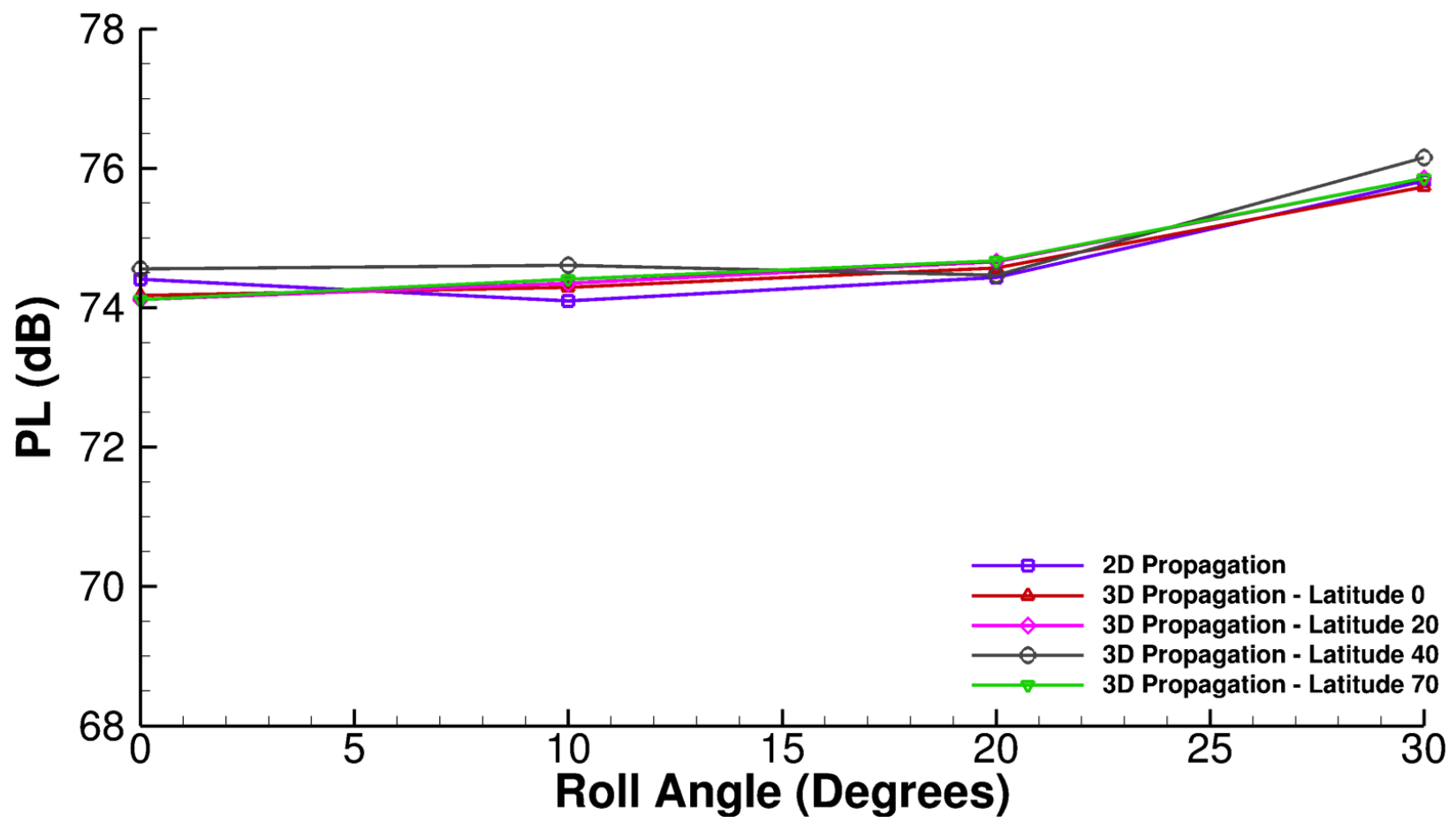

Fig. 7 Impact of ellipsoidal Earth effects on perceived loudness under zero winds

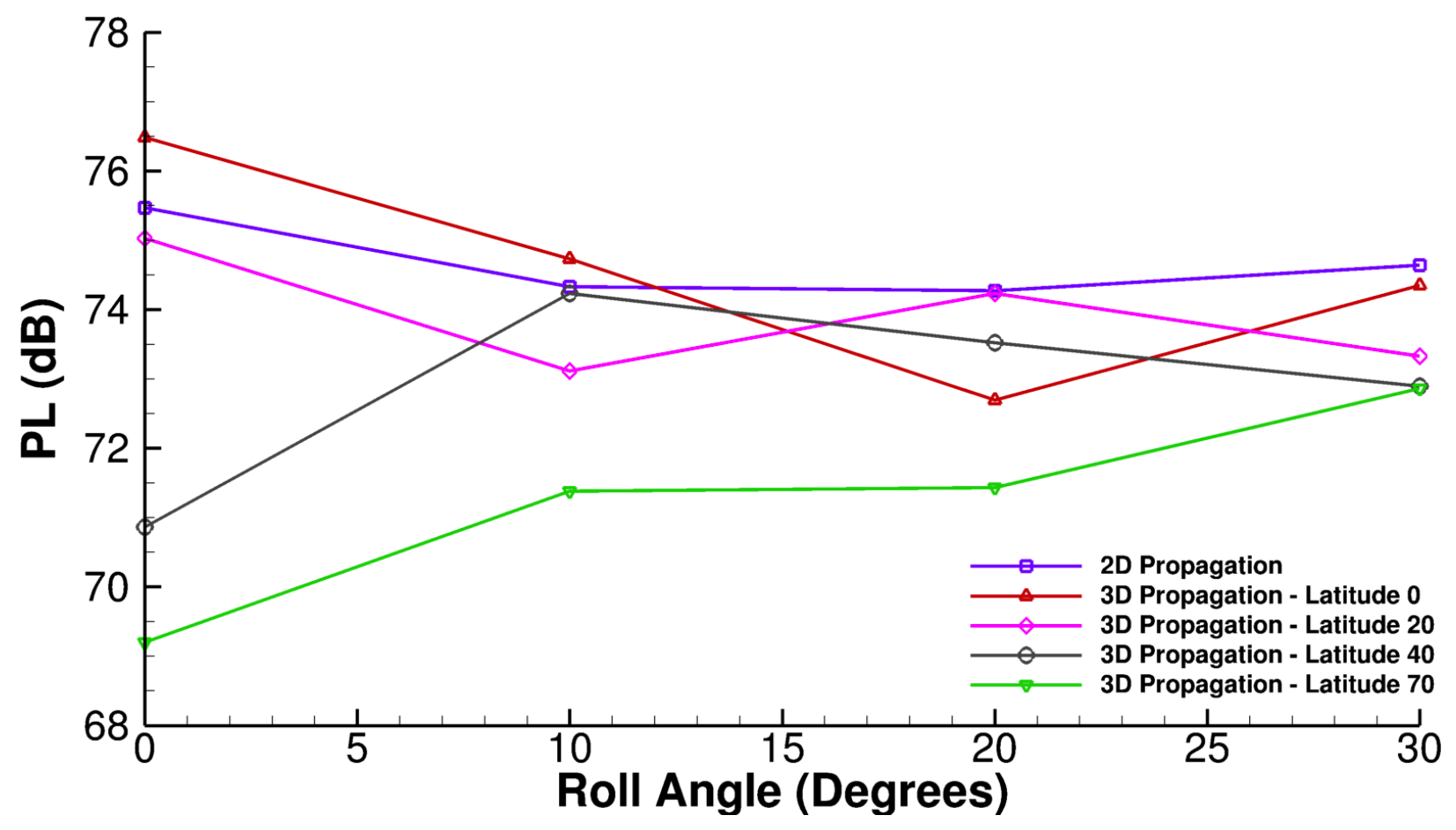

Fig. 8 Impact of ellipsoidal Earth effects on perceived loudness under arbitrary winds 


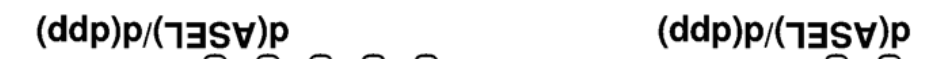

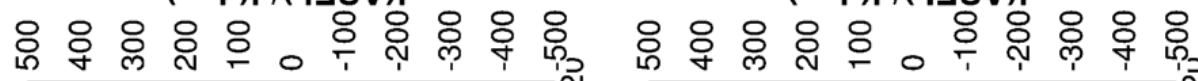

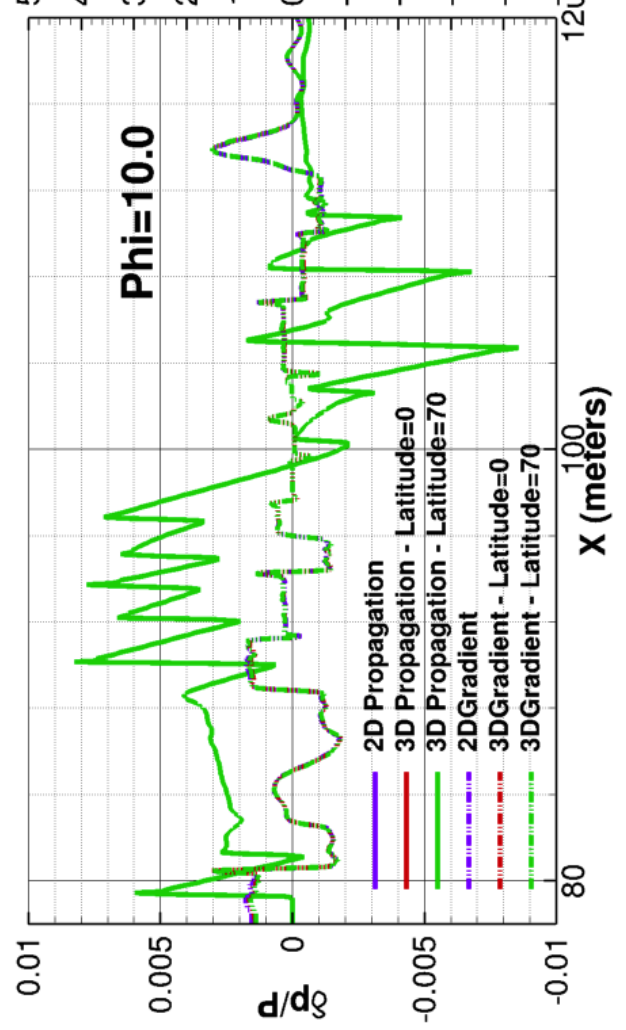

(ddp)p/(7ヨS $\forall) p$

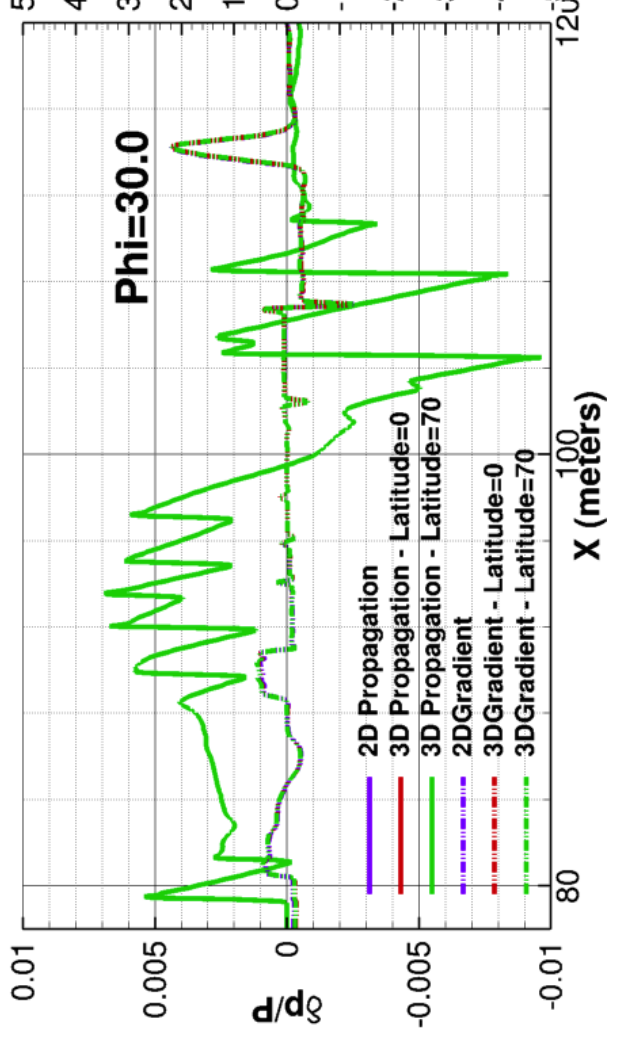

(ddp)p/(7ヨS $\forall) p$

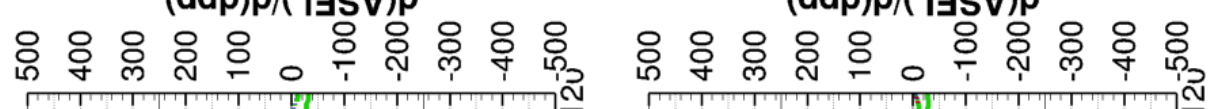
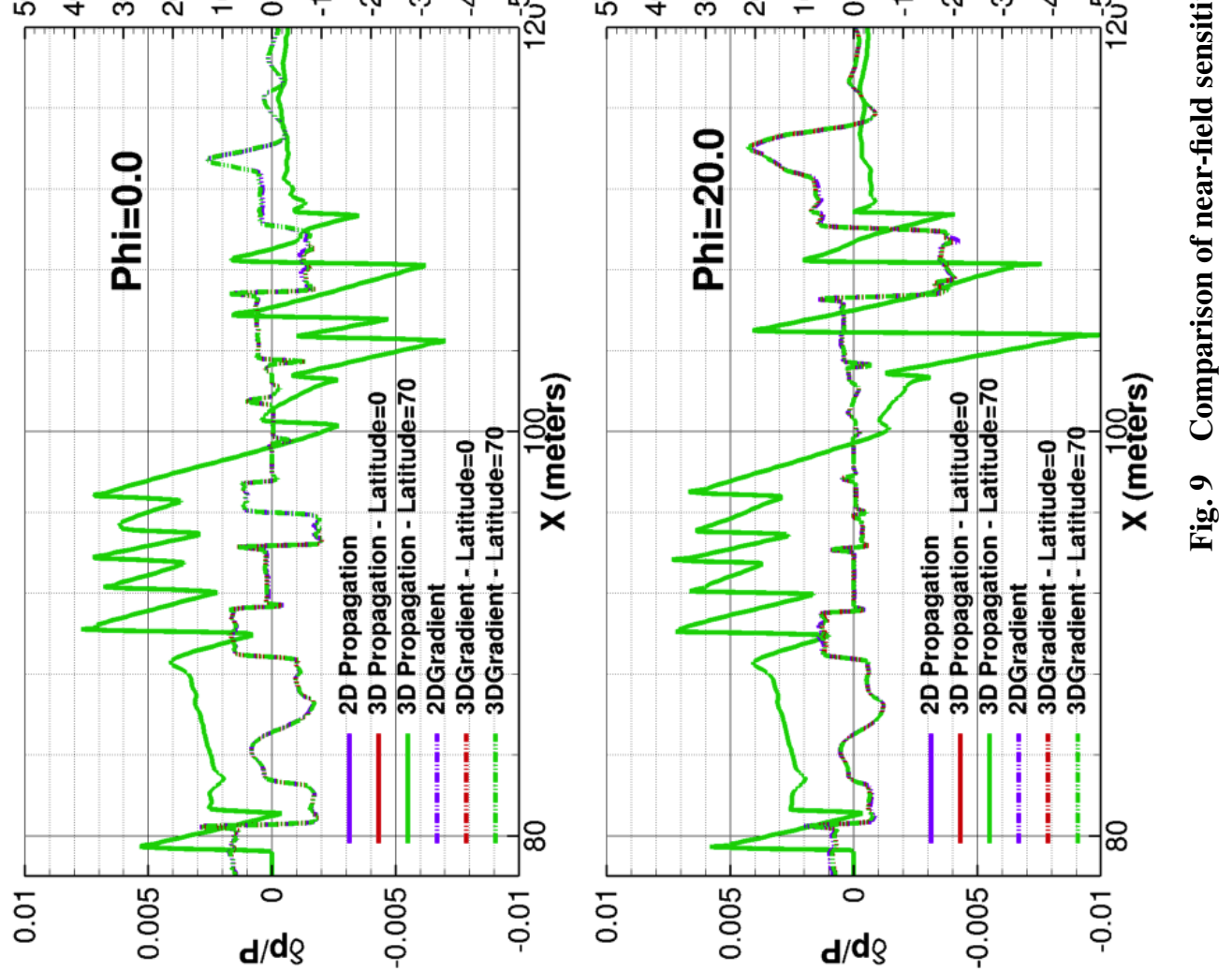


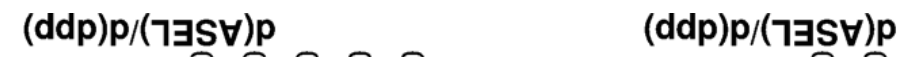

융 \&

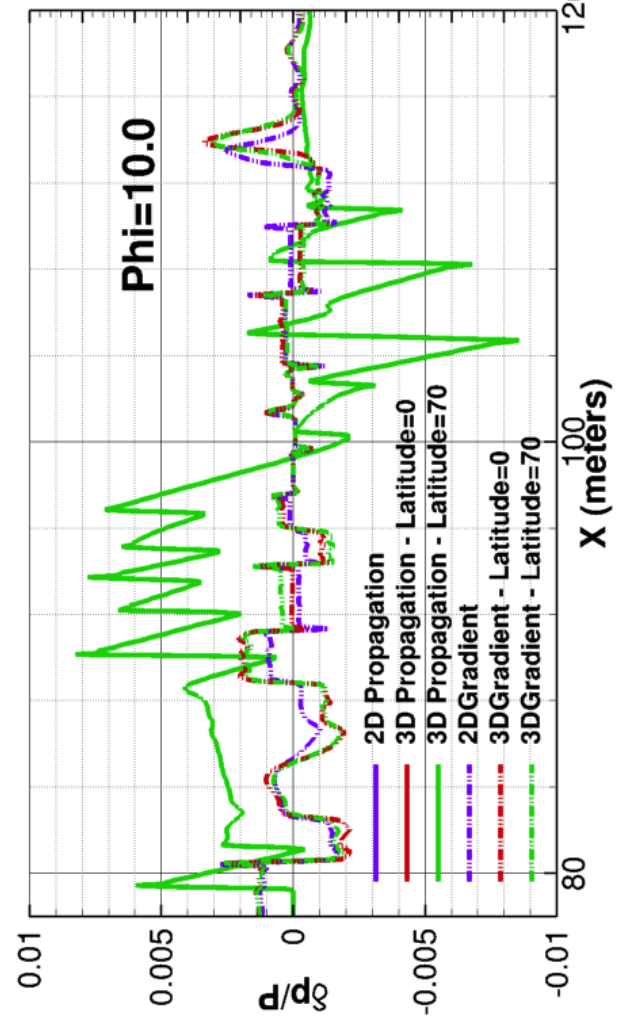

(ddp)p/(7ヨS $)$ p

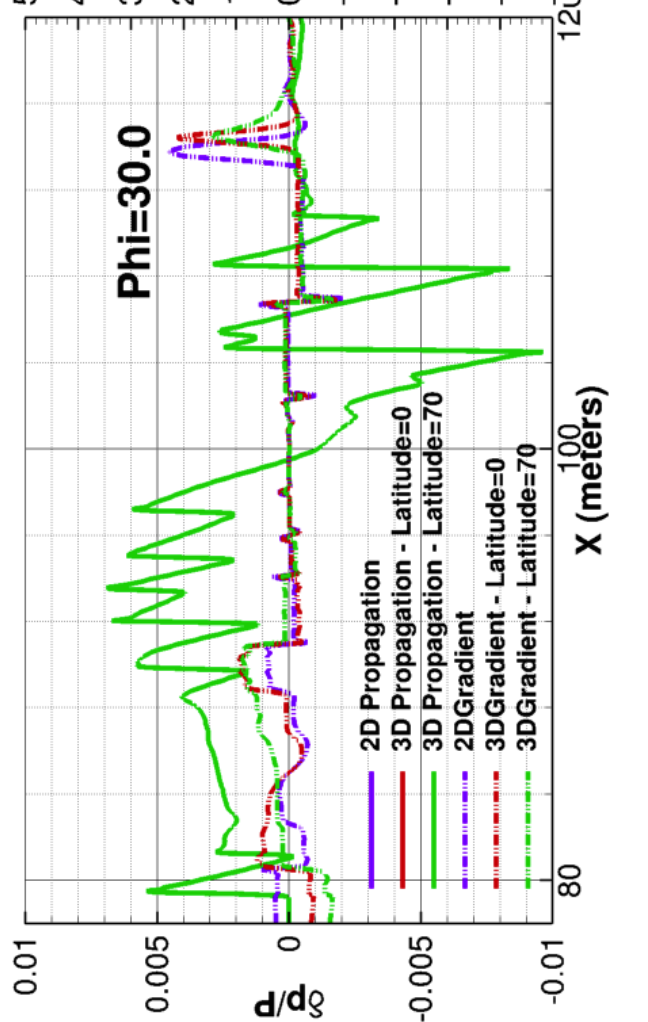

(ddp)p/(7ヨS $)$ p

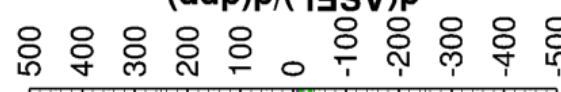
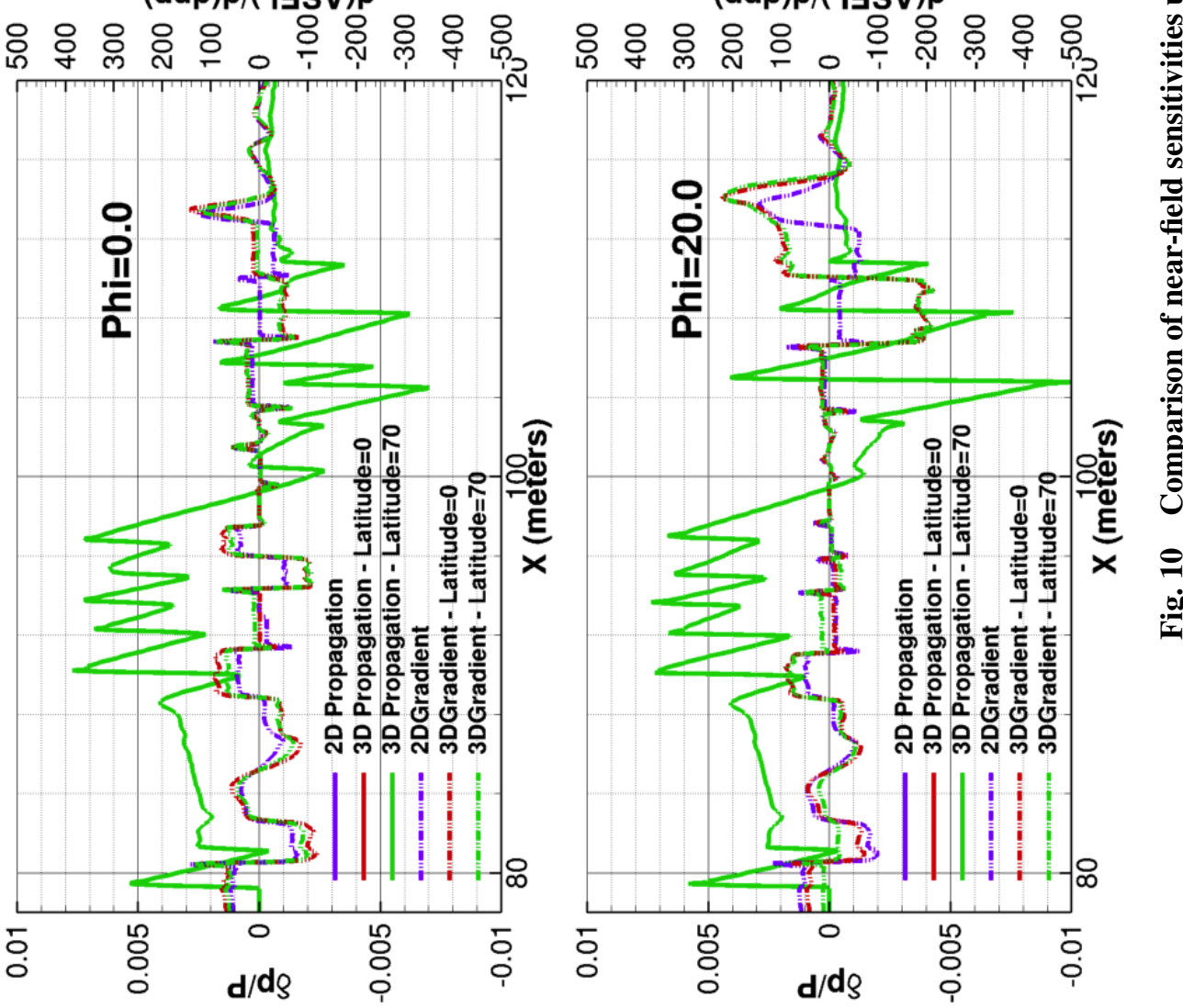

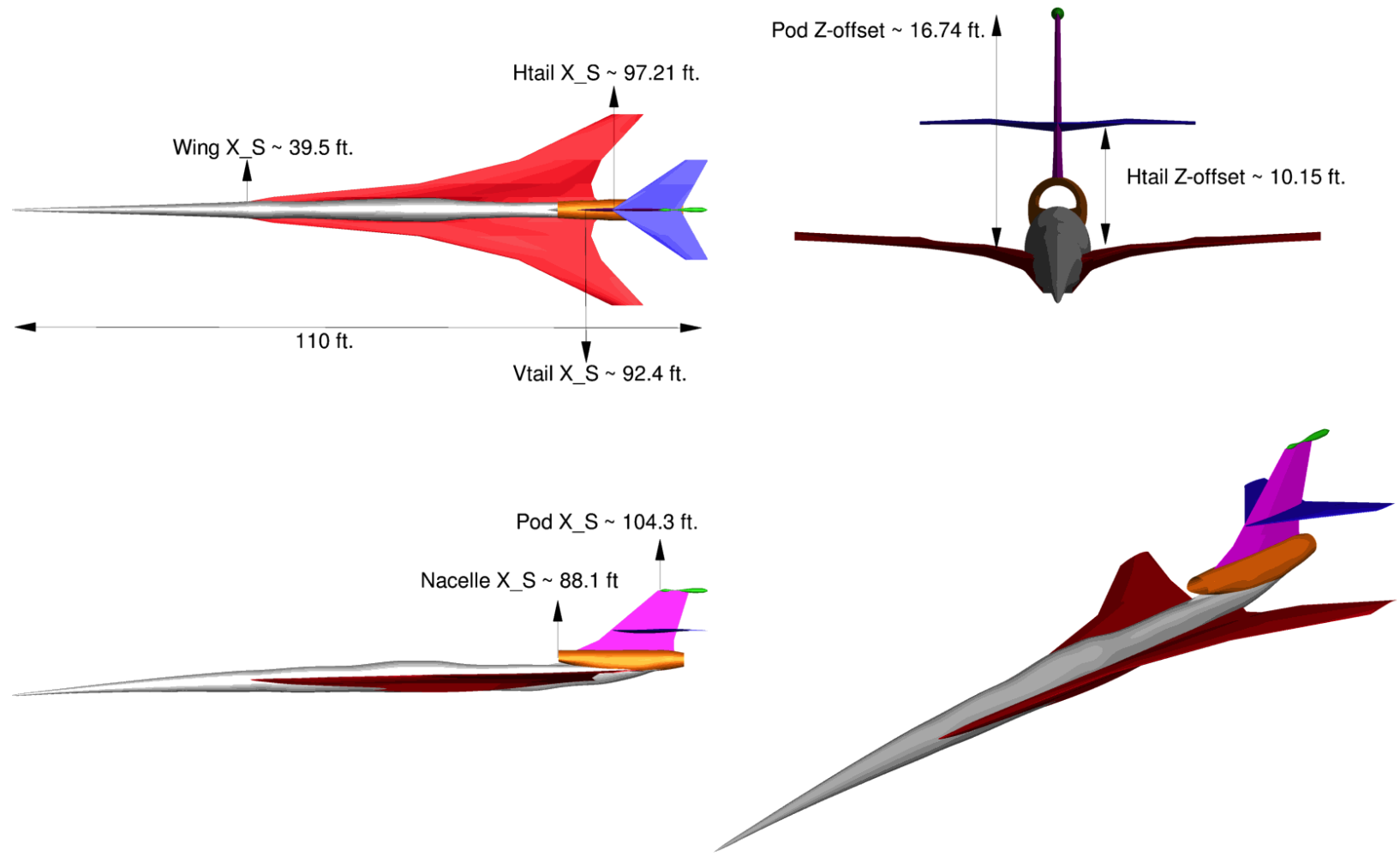

Fig. 11 Orthogonal projections of the Baseline configuration

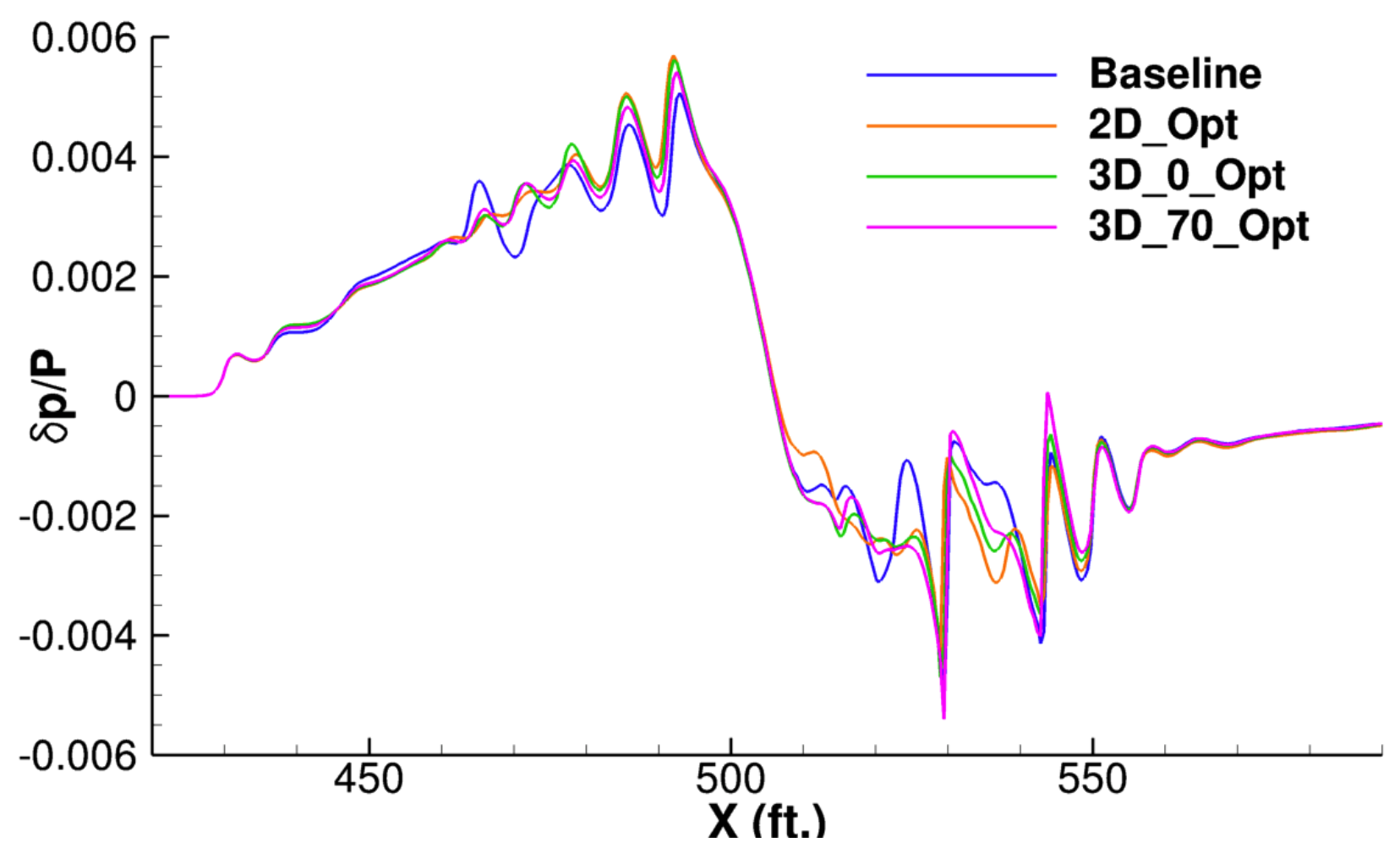

Fig. 12 Comparison of near-fields after shape optimization using flat and ellipsoidal Earth effects 


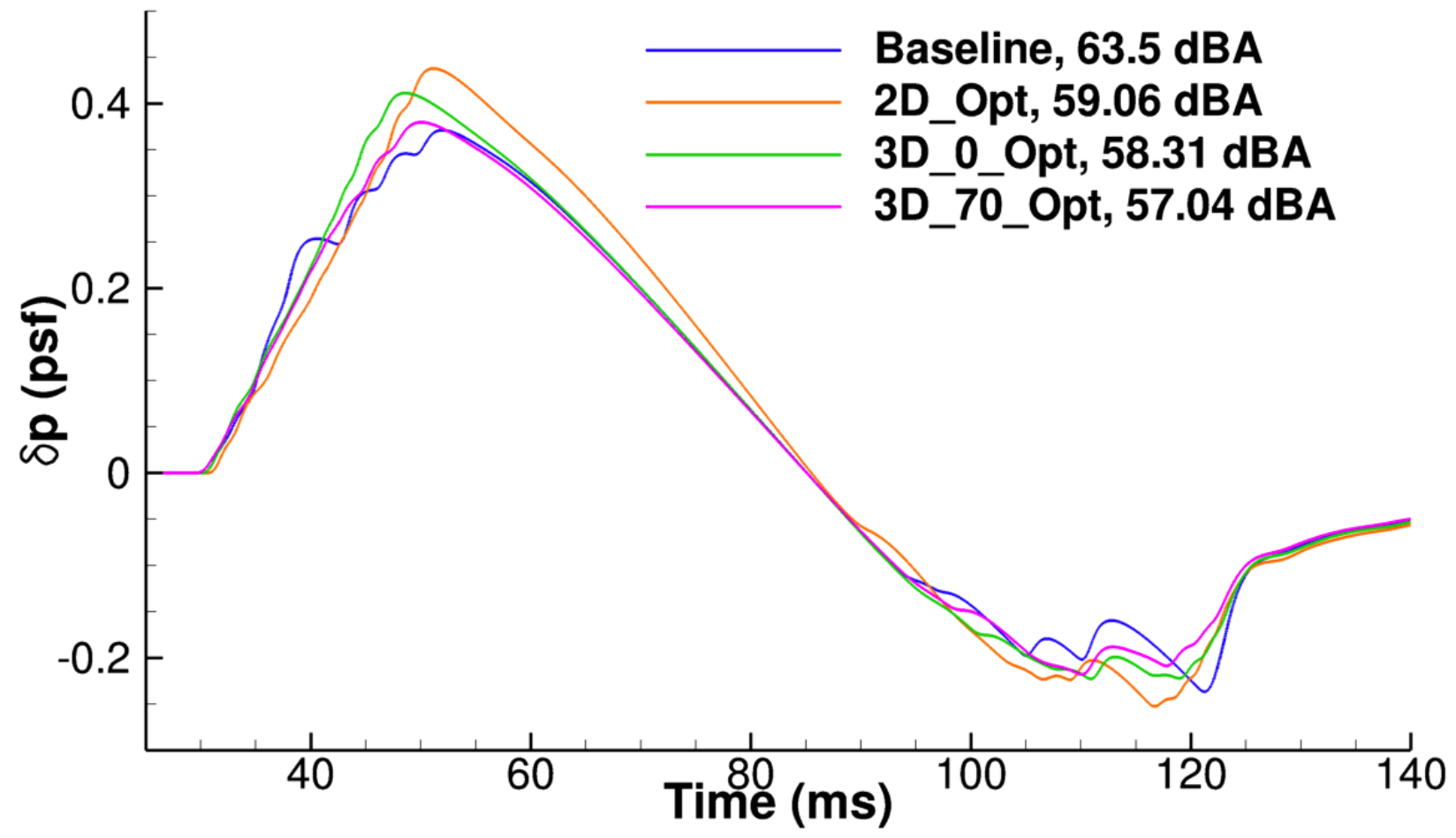

Fig. 13 Comparison of ground signatures after shape optimization using flat and ellipsoidal Earth effects

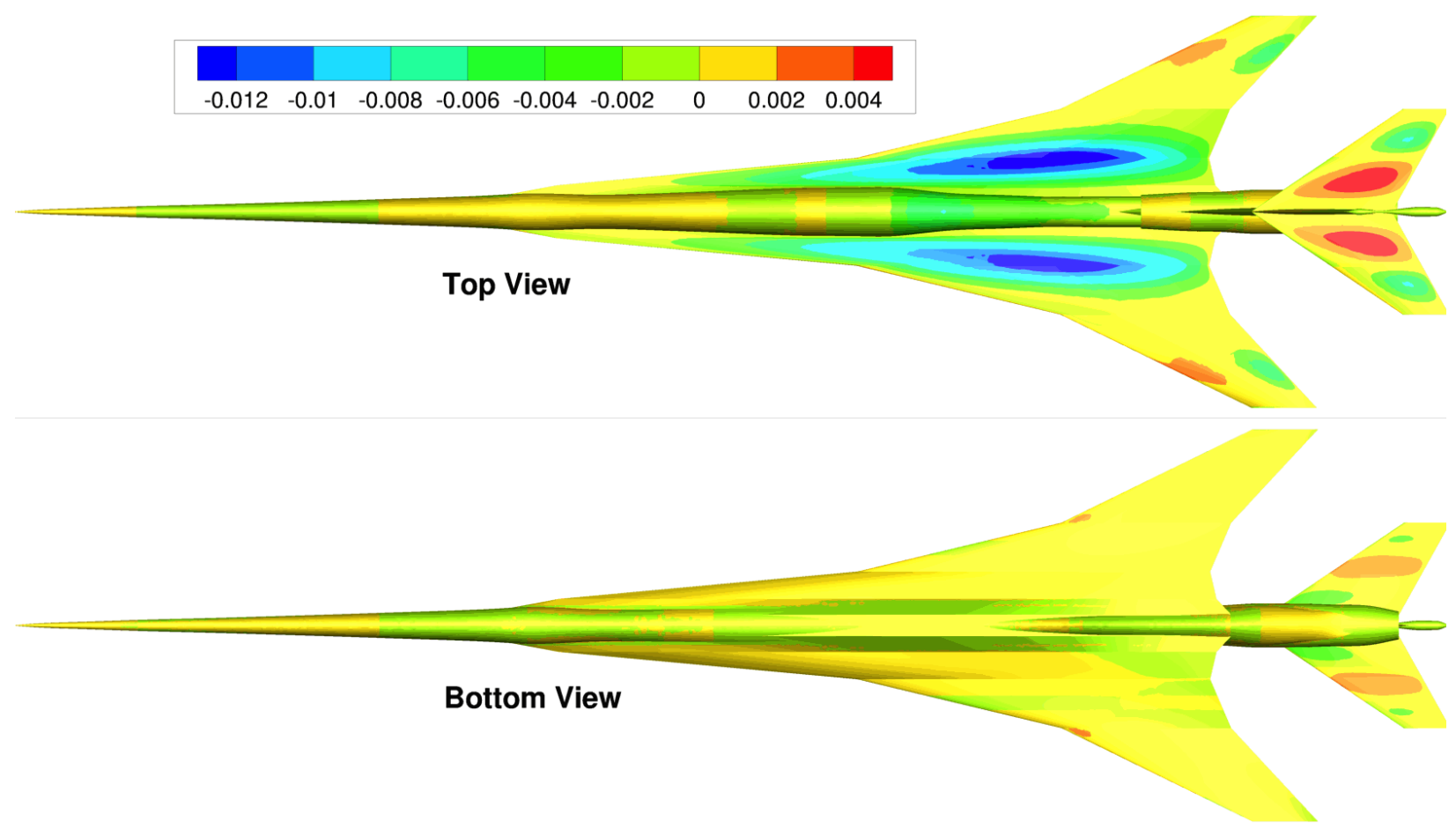

Fig. 14 Contours of normal displacement in feet of shape optimum assuming flat Earth 


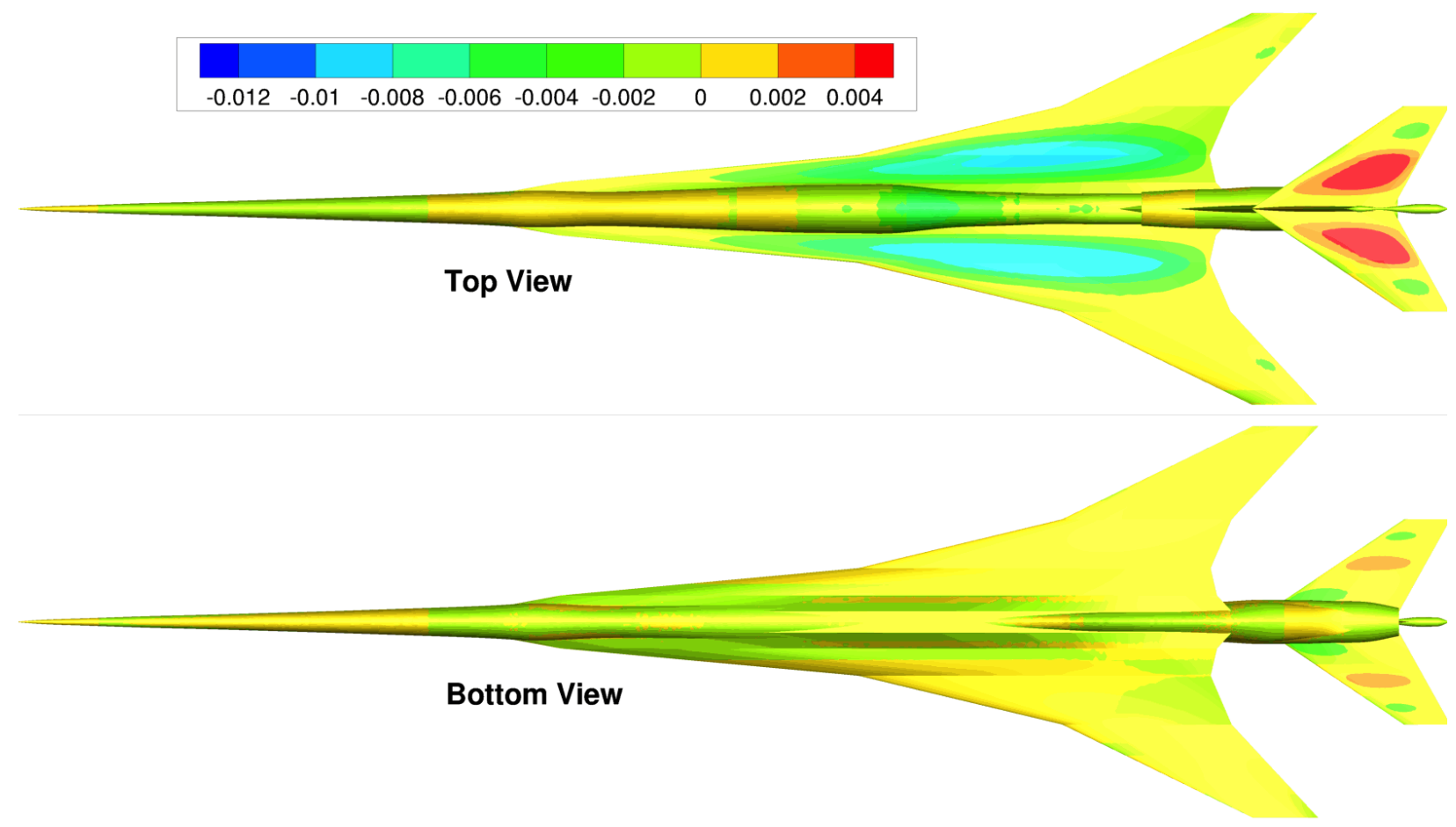

Fig. 15 Contours of normal displacement in feet of shape optimum with ellipsoidal Earth effects, Latitude $=0^{o}$

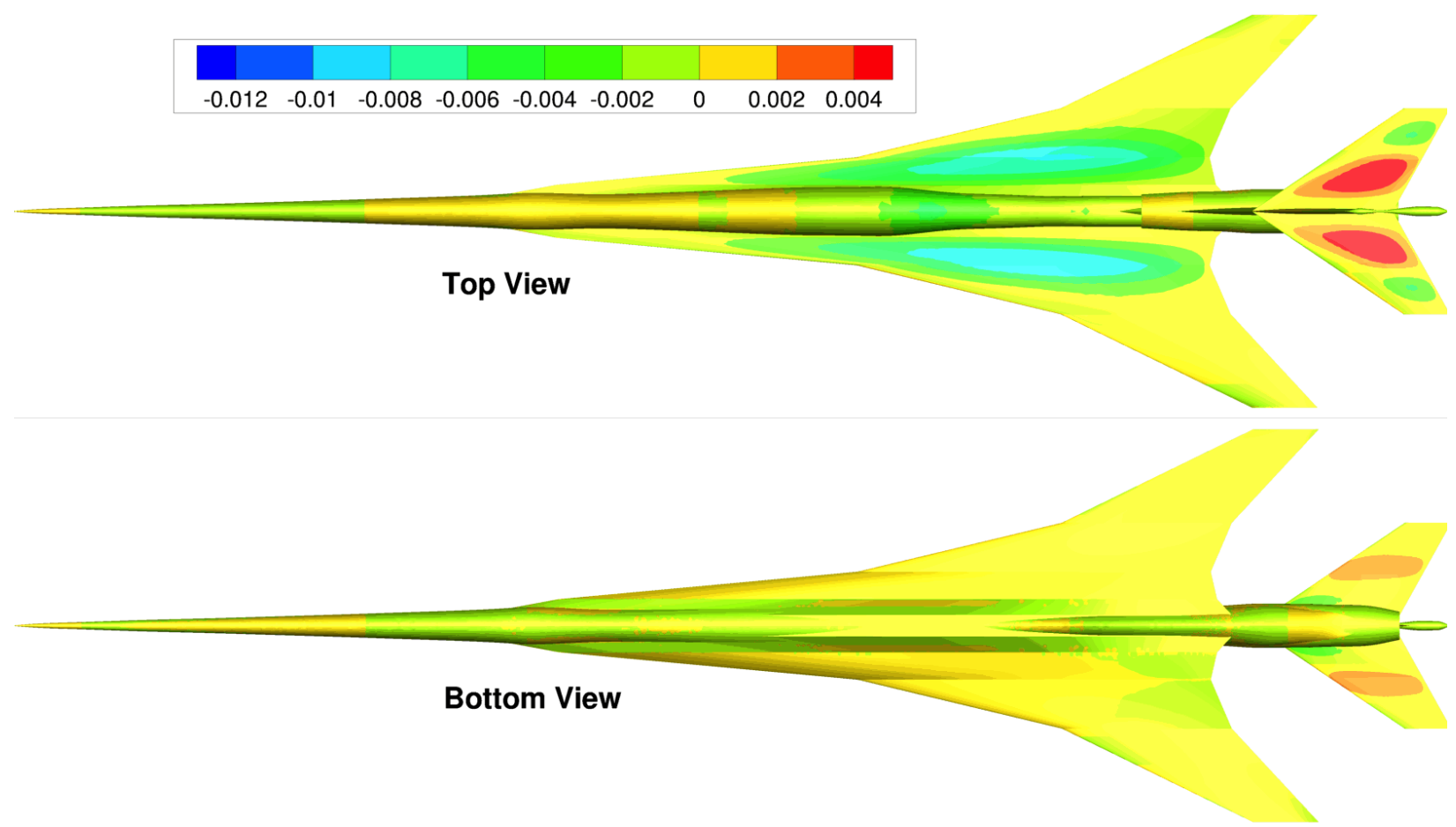

Fig. 16 Contours of normal displacement in feet of shape optimum with ellipsoidal Earth effects, Latitude $=70^{\circ}$ 

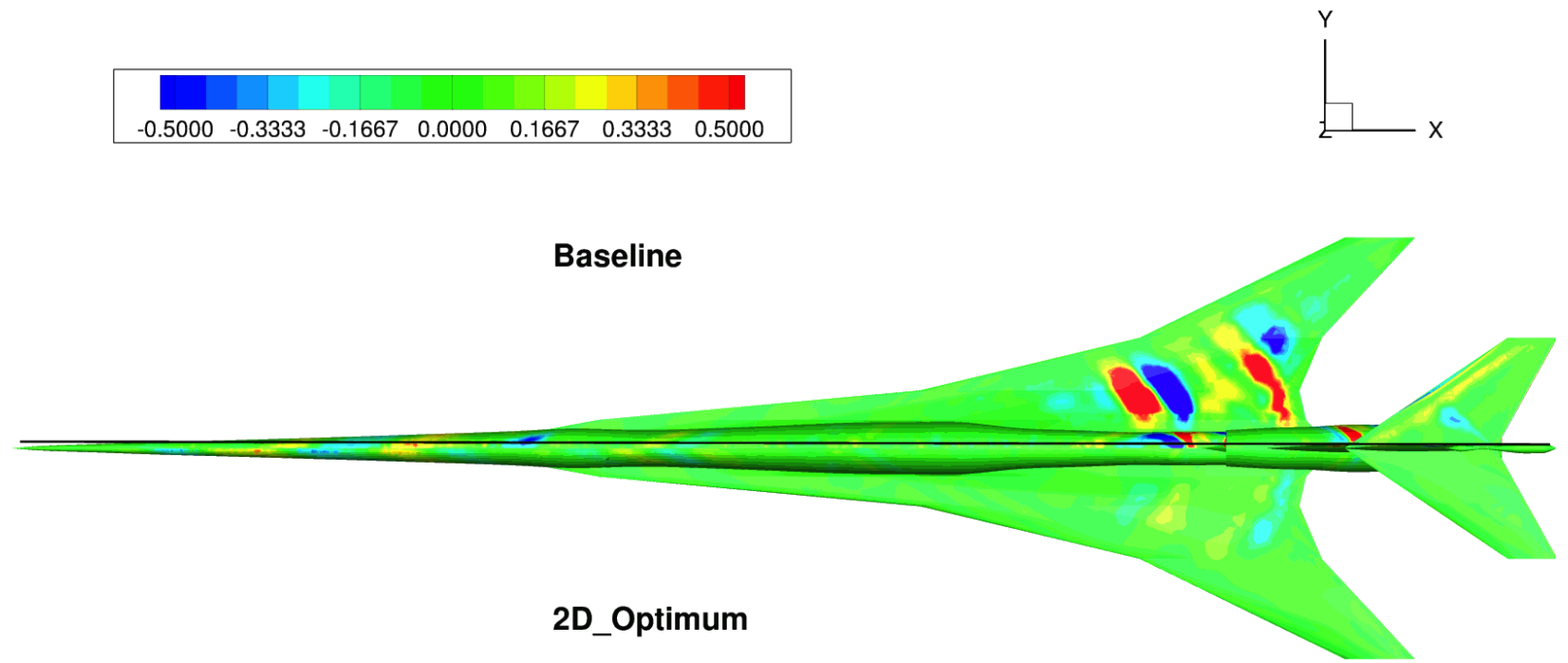

(a) Top View

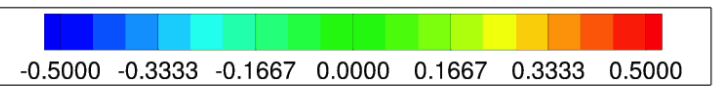

$\begin{array}{lllllll}-0.5000 & -0.3333 & -0.1667 & 0.0000 & 0.1667 & 0.3333 & 0.5000\end{array}$

2D_Optimum

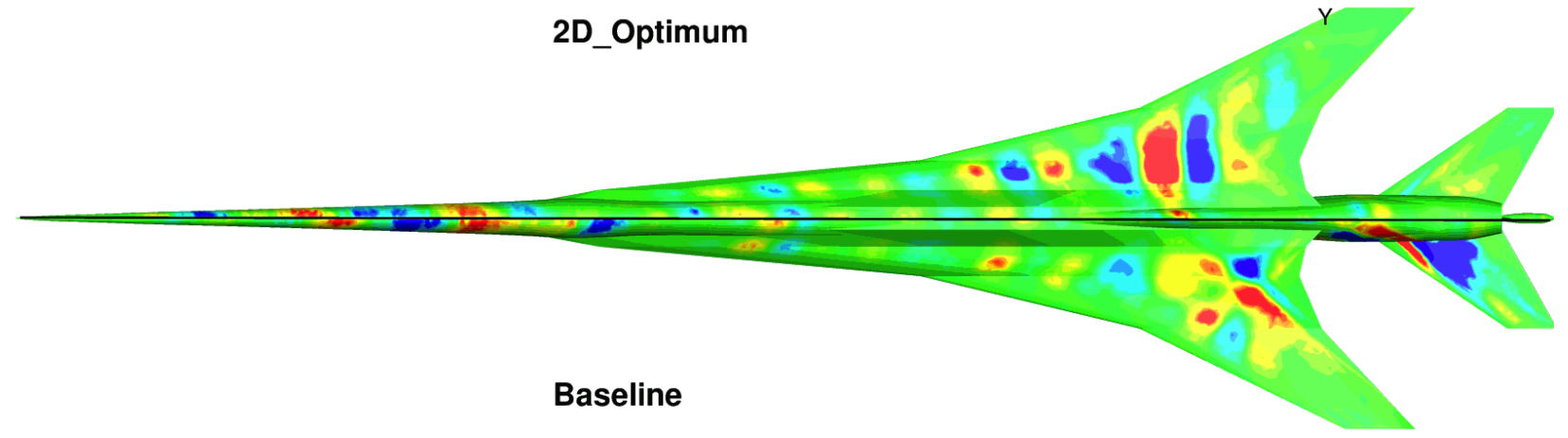

(b) Bottom View

Fig. 17 Optimum loudness sensitivity [d(ASEL)/dn, dB/feet] contours assuming flat Earth 

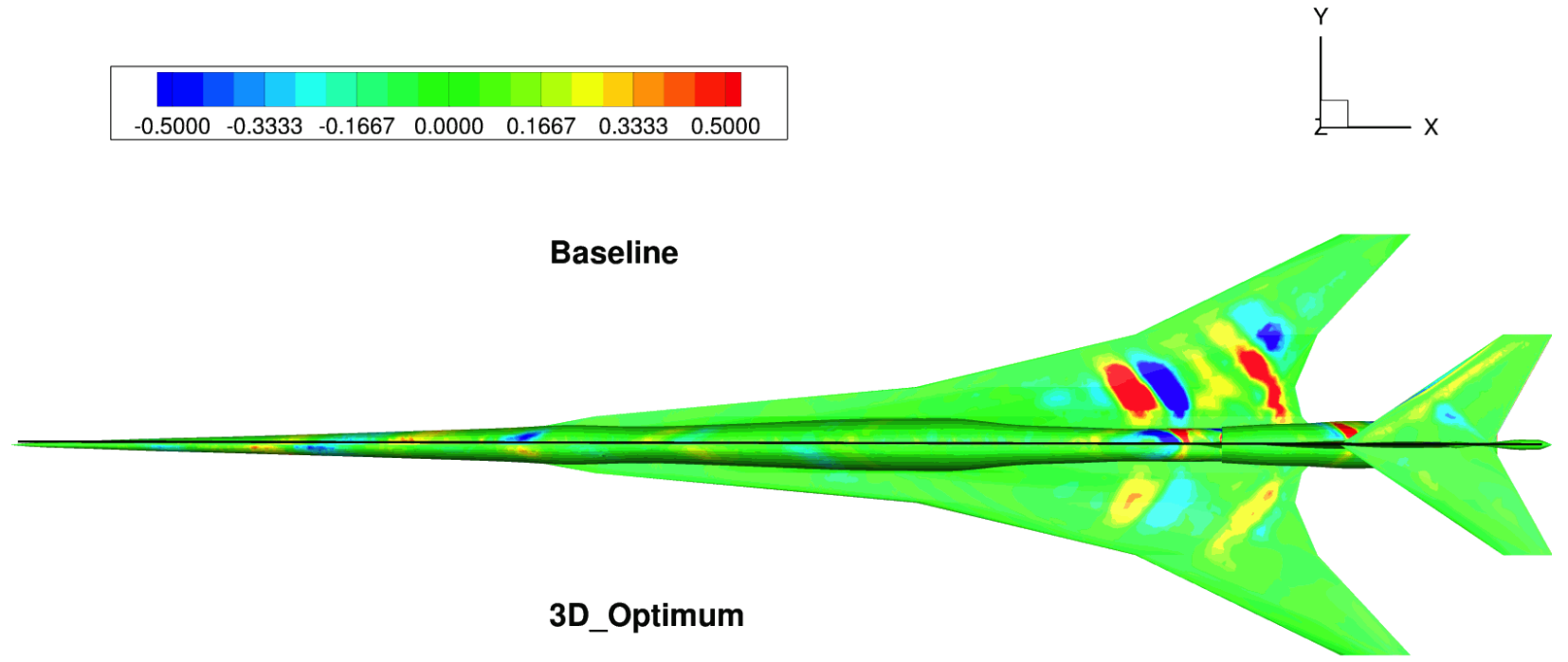

(a) Top View

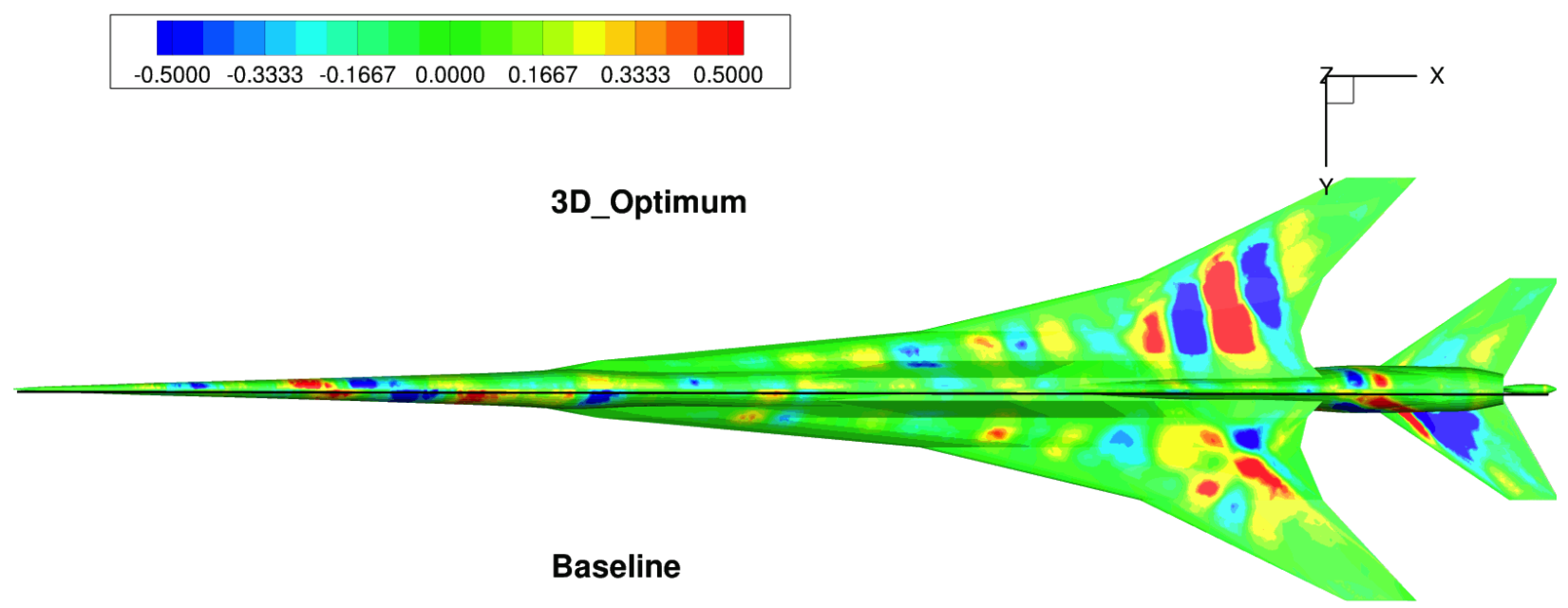

(b) Bottom View

Fig. 18 Optimum loudness sensitivity contours with ellipsoidal Earth effects, Latitude $=0^{\circ}$ 

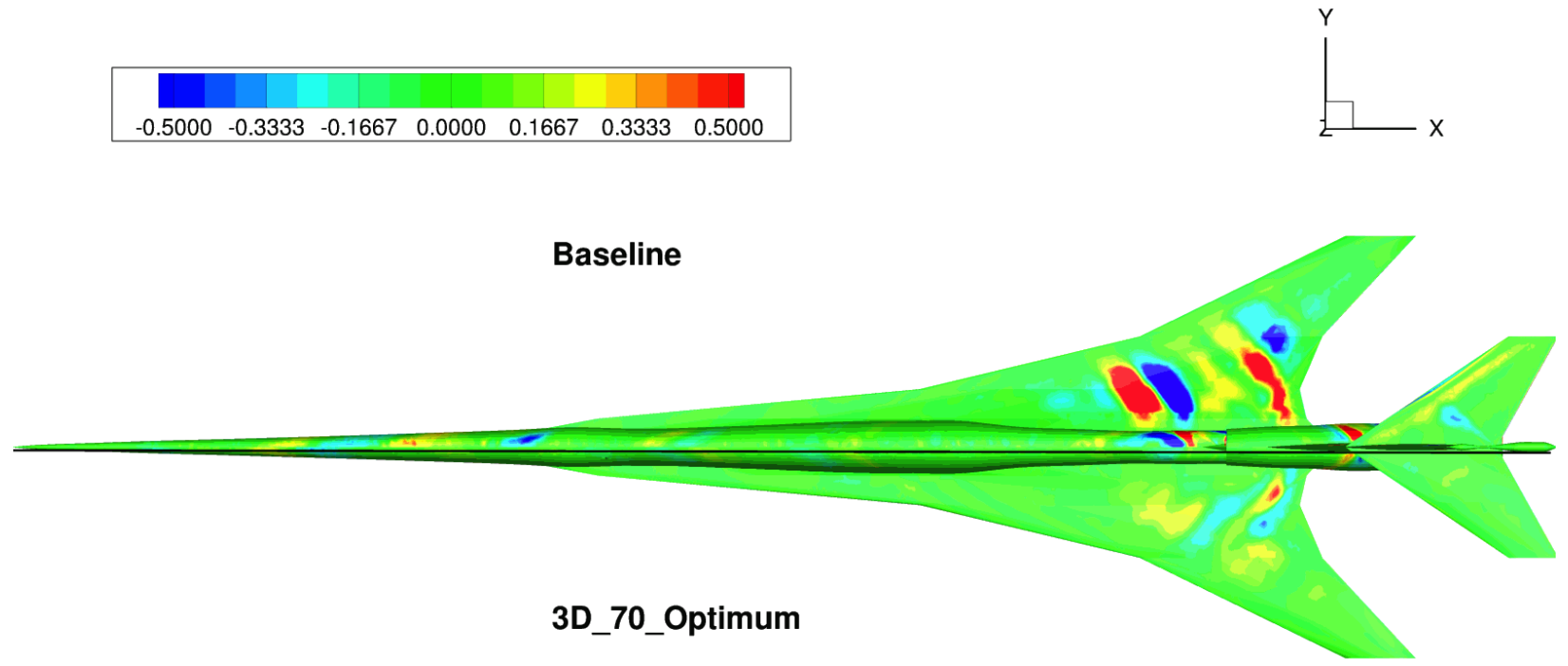

(a) Top View
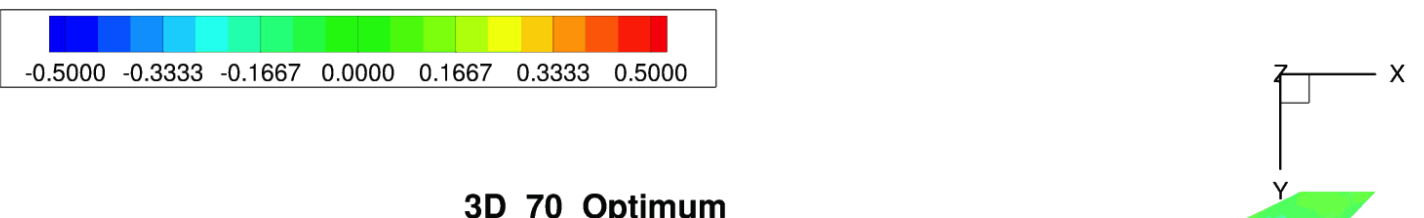

3D_70_Optimum

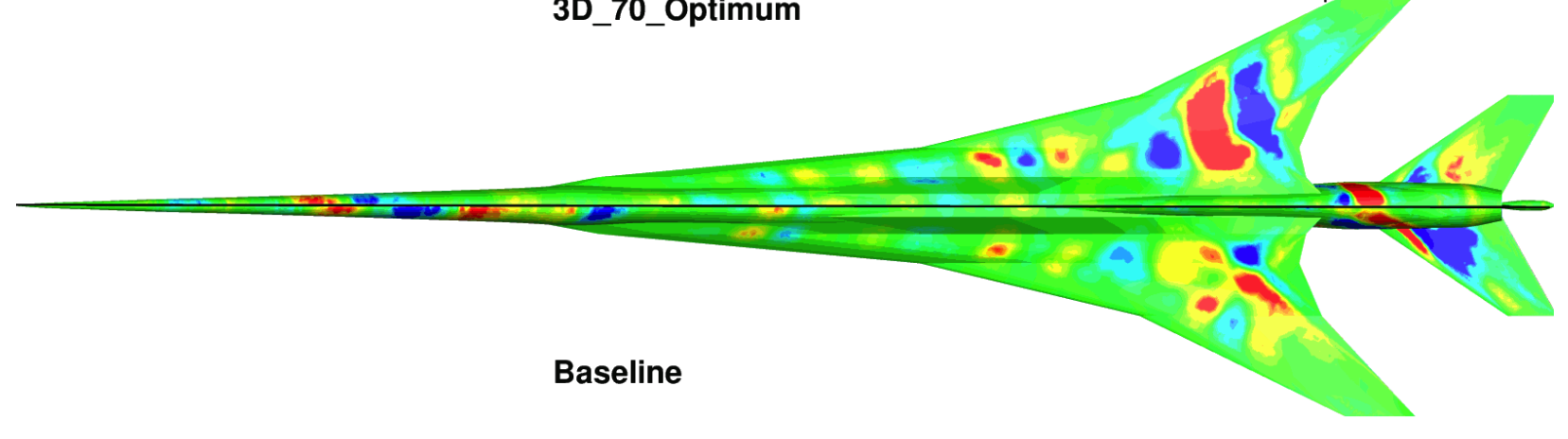

(b) Bottom View

Fig. 19 Optimum loudness sensitivity contours with ellipsoidal Earth effects, Latitude $=70^{\circ}$ 